\title{
WEIGHT FILTRATIONS VIA COMMUTING AUTOMORPHISMS
}

\author{
DANiEL R. GRAYSON \\ University of Illinois at Urbana-Champaign
}

\begin{abstract}
We consider a filtration of the $K$-theory space for a regular noetherian ring proposed by Goodwillie and Lichtenbaum and show that its successive quotients are geometric realizations of explicit simplicial abelian groups. The filtration in weight $t$ involves $t$-tuples of commuting automorphisms of projective $R$-modules. It remains to show that the Adams operations act appropriately on the filtration.
\end{abstract}

\section{Introduction.}

Much of the interest in algebraic $K$-theory today arises because it points the way toward development of a motivic cohomology theory. According to the philosophy of Beilinson, if $R$ is a regular noetherian ring and $X=\operatorname{Spec}(R)$, then motivic cohomology groups $H^{m}(X, \mathbb{Z}(t))$, once defined, ought to appear in a spectral sequence

$$
E_{2}^{p q}=H^{p-q}(X, \mathbb{Z}(-q)) \Rightarrow K_{-p-q}(R)
$$

which is reminiscent of the spectral sequence of Atiyah and Hirzebruch for topological $K$-theory. A natural way to approach this problem is to take the space $K(R)$ whose homotopy groups are the $K$-groups, $K_{n}(R)=\pi_{n} K(R)$, and produce a filtration

$$
K(R)=W^{0} \leftarrow W^{1} \leftarrow W^{2} \leftarrow \ldots
$$

of it. See, for example, the exposition in [9]. The two basic requirements are

(A) that the quotient $W^{t} / W^{t+1}$ should be the geometric realization of a simplicial abelian group, and

(B) that the Adams operations $\psi^{k}$ act on the filtration, and act purely on the quotients $W^{t} / W^{t+1}$, i.e., by multiplication by $k^{t}$.

Adopting the definition $\mathbb{Z}(t)=\Omega^{2 t} W^{t} / W^{t+1}$ in the sense of spectra (with negative homotopy groups), or equivalently $H^{m}(X, \mathbb{Z}(t))=\pi_{2 t-m}\left(W^{t} / W^{t+1}\right)$, leads immediately to an exact couple and thus to a spectral sequence as in (1.1), and allows one (if the two basic requirements are fulfilled) to identify $H^{m}(X, \mathbb{Q}(t))$ with $K_{2 t-m}(R)_{\mathbb{Q}}^{(t)}$, the weight $t$ part of $K_{2 t-m}(R)_{\mathbb{Q}}$.

The Postnikov filtration of $K(R)$ is an example where (A) is fulfilled, but (B) is not. The quotients are geometric realizations of simplicial abelian groups with a single

1991 Mathematics Subject Classification. Primary 19E20.

Supported by NSF grant DMS 90-02715. I thank Tom Goodwillie, Stephen Lichtenbaum, Friedhelm Waldhausen, Steven Landsburg, and Stephen Ullom for useful discussions and ideas. 
nonvanishing homotopy group, but they fail to have pure weight, because $K_{n} R$ is not pure of weight $n$, even for $n=3$ and $R$ a field.

In this paper we examine a proposed filtration $W^{t}$ of $K(R)$ due to Goodwillie and Lichtenbaum. In section 2 we review the motivation behind their proposal, which makes property (B) plausible. We prove that each quotient $W^{t} / W^{t+1}$ is the geometric realization of a simplicial abelian group (10.6), thereby fulfilling requirement (A). This provides the first known example of a filtration of $K(R)$ satisfying (A), and which could conceivably satisfy (B), at least when $R$ is local. The fact that the simplicial abelian group is known explicitly may make further computations possible.

\section{The proposed filtration.}

I explain now the ideas of Goodwillie and Lichtenbaum.

Goodwillie has made the following important motivational observation. Suppose the filtration $W^{t}$ is compatible with multiplication, in the sense that there are pairings $W^{i} \wedge W^{j} \rightarrow W^{i+j}$. Suppose also that $W^{0} / W^{1} \cong \mathbb{Z}$; according to property (B), we expect this when $R$ is a domain because the weight zero part of $K$-theory all occurs in $K_{0}$. It follows that $W^{i} / W^{i+1}$ will be a module for the ring $W^{0} / W^{1} \cong \mathbb{Z}$. Hence $W^{i} / W^{i+1}$ will be homotopy equivalent to the geometric realization of a simplicial abelian group, by a general fact about spectra which are $\mathbb{Z}$-modules.

In order to arrive at the proposed definition of $W^{t}$, let us consider temporarily the case where $R$ is local and see what advantages arise in that case. Soulé has shown [16, Corollary 1, p. 498] for such local rings that

$$
K_{n}(R)_{\mathbb{Q}}^{(t)}=0 \quad \text { for } n<t
$$

Thus we might well expect that the homotopy groups

$$
\pi_{0} W^{t}, \pi_{1} W^{t}, \ldots, \pi_{t-1} W^{t}
$$

would vanish, and that $W^{t}$ would arise as the $t$-fold delooping of another space $V^{t}$. The group $\pi_{0} V^{t}=\pi_{t} W^{t}$ would be related to the weight $t$ part of $K_{t}(R)$, which in turn is closely related to the Milnor $K$-group $K_{t}^{M}(R)$. The Milnor $K$-group is constructed from $t$-tuples of units in $R$, so the space $V^{t}$ might be constructed from the same ingredients, but it is a bit hard to see how to extract homotopy theory from units. The idea of Goodwillie and Lichtenbaum is to use invertible matrices (or, equivalently, automorphisms of projective modules) instead.

When $\mathcal{M}$ is an exact category we let $K \mathcal{M}$ denote the space whose homotopy groups are the $K$-groups, i.e., $K_{n} \mathcal{M}=\pi_{n} K \mathcal{M}$. Given a ring $R$ let $\mathcal{P}(R)$ denote the exact category of finitely generated projective $R$-modules. Given two rings $R$ and $S$ with 1 , let $\mathcal{P}(R, S)$ denote the exact category of $R$-S-bimodules which as $R$-modules are finitely generated and projective. Observe that $\mathcal{P}(R, \mathbb{Z})=\mathcal{P}(R)$. Set $K(R, S)=K \mathcal{P}(R, S)$. If $T$ is another ring, then tensor product over $S$ gives a bi-exact functor $\mathcal{P}(R, S) \times$ $\mathcal{P}(S, T) \rightarrow \mathcal{P}(R, T)$ which leads to a pairing $K(R, S) \wedge K(S, T) \rightarrow K(R, T)$. Let $\mathbb{G}_{m}$ denote the multiplicative group, as a group scheme over $\operatorname{Spec}(\mathbb{Z})$. The underlying scheme is $\mathbb{G}_{m}=\operatorname{Spec} \mathbb{Z}\left[U, U^{-1}\right]$. We see that the exact category $\mathcal{P}\left(R, \mathbb{G}_{m}\right)=\mathcal{P}\left(R, \mathbb{Z}\left[U, U^{-1}\right]\right)$ is (isomorphic to) the exact category where an object is a pair $(P, \theta)$ consisting of a finitely 
generated projective $R$-module $P$ and an automorphism $\theta \in \operatorname{Aut}(P)$. This is the same category that was considered in [5] and [6].

We can generalize this notation a bit: if $\mathcal{M}$ is an exact category, and $S$ is a ring, then we let $\mathcal{M}(S)$ denote the exact category of pairs $(M, \rho)$, where $M$ is an object of $\mathcal{M}$ and $\rho: S \rightarrow \operatorname{End}_{\mathcal{M}} M$ is an "S-module structure" for $M$. As above, we may interpret $\mathcal{M}\left(\mathbb{G}_{m}\right)$ as the exact category of automorphisms of objects of $\mathcal{M}$. Observe also that $\mathcal{P}(R)(S)=\mathcal{P}(R, S)$. This definition has often been used before, and appears in [14, 12.1-2] and [11].

The pairing $K\left(R, \mathbb{G}_{m}\right) \wedge K\left(\mathbb{G}_{m}\right) \rightarrow K(R)$ together with multiplication by the unit $U \in \mathbb{Z}\left[U, U^{-1}\right]^{\times} \cong K_{1} \mathbb{Z}\left[U, U^{-1}\right]$ gives a map $K\left(R, \mathbb{G}_{m}\right) \rightarrow \Omega K(R)$, which is presumably the same as the map considered in [5] and [6]. On homotopy groups, the image of that map ought to consist of elements of weight $\geq 1$, because $U$, being a unit, has pure weight 1 , and weights add upon multiplication. Since 1 is the trivial element of the group $K_{1} \mathbb{G}_{m}$, multiplication by $U$ kills the $K$-theory of the subcategory $\mathcal{P}(R, 1)$ of $\mathcal{P}\left(R, \mathbb{G}_{m}\right)$ consisting of those pairs $(P, \theta)$ where $\theta$ is the identity. Let's introduce $K\left(R, \mathbb{G}_{m}^{\wedge 1}\right)$ as notation for the second factor in the decomposition $K\left(R, \mathbb{G}_{m}\right) \sim K(R) \times K\left(R, \mathbb{G}_{m}{ }^{\wedge 1}\right)$. The relevant part of the map above is the map $K\left(R, \mathbb{G}_{m}^{\wedge 1}\right) \rightarrow \Omega K(R)$.

Similarly, we may consider $\mathcal{P}\left(R, \mathbb{G}_{m}{ }^{t}\right)$, each of whose objects $\left(P, \theta_{1}, \ldots, \theta_{t}\right)$ is essentially a finitely generated projective $R$-module $P$ equipped with commuting automorphisms $\theta_{1}, \ldots, \theta_{t}$. Multiplication by $U_{t}$ gives us a map $K\left(R, \mathbb{G}_{m}{ }^{t}\right) \rightarrow \Omega K\left(R, \mathbb{G}_{m}{ }^{t-1}\right)$, and multiplication by $U_{1} \cdot \ldots \cdot U_{t}$ gives us a map $K\left(R, \mathbb{G}_{m}{ }^{t}\right) \rightarrow \Omega^{t} K(R)$ whose image on homotopy groups ought to consist of elements of weight $\geq t$. The latter map dies on each of the subcategories $\mathcal{P}\left(R, \mathbb{G}_{m} \times \cdots \times 1 \times \cdots \times \mathbb{G}_{m}\right)$ where one of the automorphisms is the identity, and indeed it factors through the iterated cofiber space constructed from the $t$-dimensional cube of spaces obtained from $K\left(R, \mathbb{G}_{m}{ }^{t}\right)$ by setting various $\mathbb{G}_{m}$ factors to 1 .

Here is some convenient notation for cubes, as in $[8, \S 4]$. We let [1] denote the ordered set $\{0<1\}$ regarded as a category, and we use $\epsilon$ as notation for an object of [1]. By an $n$-dimensional cube in a category $\mathcal{C}$ we will mean a functor from $[1]^{n}$ to $\mathcal{C}$. An object $\mathrm{C}$ in $\mathcal{C}$ gives a 0 -dimensional cube denoted by $[C]$, and an arrow $C \rightarrow C^{\prime}$ in $\mathcal{C}$ gives a 1 -dimensional cube denoted by $\left[C \rightarrow C^{\prime}\right]$. If the category $\mathcal{C}$ has products, we may define an external product of cubes as follows. Given an $n$-dimensional cube $X$ and an $n^{\prime}$-dimensional cube $Y$ in $\mathcal{C}$, we let $X \otimes Y$ denote the $n+n^{\prime}$-dimensional cube defined by $(X \otimes Y)\left(\epsilon_{1}, \ldots, \epsilon_{n+n^{\prime}}\right)=X\left(\epsilon_{1}, \ldots, \epsilon_{n}\right) \times Y\left(\epsilon_{n+1}, \ldots, \epsilon_{n+n^{\prime}}\right)$. If $\mathcal{M}$ is an exact category, and $X$ is a cube of affine schemes, then we let $\mathcal{M}(X)$ denote the cube of exact categories defined by $\mathcal{M}(X)\left(\epsilon_{1}, \ldots, \epsilon_{n}\right)=\mathcal{M}\left(X\left(\epsilon_{1}, \ldots, \epsilon_{n}\right)\right)$. Let $\mathbb{G}_{m}^{\wedge t}$ denote the external product of $t$ copies of $\left[1 \rightarrow \mathbb{G}_{m}\right]$. The cube $\mathbb{G}_{m}^{\wedge t}$ of affine schemes gives rise to a cube $\mathcal{P}\left(R, \mathbb{G}_{m}{ }^{\wedge t}\right)=\mathcal{P}(R)\left(\mathbb{G}_{m}{ }^{\wedge t}\right)$ of exact categories. In [8, §4] is presented a construction called $C$ which can be applied to a cube of exact categories to convert it into a multisimplicial exact category, the $K$-theory of which serves as the iterated cofiber space of the corresponding cube of $K$-theory spaces. We use $K\left(R, \mathbb{G}_{m}{ }^{\wedge t}\right)$ as notation for that $K$-theory space, and we let $K_{n}\left(R, \mathbb{G}_{m}^{\wedge t}\right)=\pi_{n} K\left(R, \mathbb{G}_{m}^{\wedge t}\right)$. We see that $K_{0}\left(R, \mathbb{G}_{m}^{\wedge t}\right)$ can be described more concretely as the quotient of $K_{0}\left(R, \mathbb{G}_{m}{ }^{t}\right)$ by the subgroup generated by those objects $\left(P, \theta_{1}, \ldots, \theta_{t}\right)$ where $\theta_{i}=1$ for some $i$.

We let Ord denote the category of finite nonempty ordered sets, and for each $d \geq 0$ we introduce the object $[d]=\{0<1<\cdots<d\}$ of Ord. We let $\Delta^{d}=\operatorname{Hom}_{\text {Ord }}(-,[d])$, 
so that $\left|\Delta^{d}\right|$ is the standard $d$-simplex.

We recall now the standard simplicial polynomial ring over $R$, which occurs in the Karoubi-Villamayor $K$-theory. We introduce the affine space $\mathbb{A}^{d}$ of dimension $d$ with the coordinate system implied by writing it as

$$
\mathbb{A}^{d}=\operatorname{Spec}\left(\mathbb{Z}\left[T_{0}, \ldots, T_{d}\right] /\left(T_{0}+\cdots+T_{d}-1\right) .\right.
$$

For $d \geq 0$ one has the $R$-algebra

$$
R \mathbb{A}^{d}=R\left[T_{0}, \ldots, T_{d}\right] /\left(T_{0}+\cdots+T_{d}-1\right) .
$$

It is a polynomial ring over $R$ in $d$ variables, and is isomorphic to $R$ when $d=0$. Given a map $[d] \stackrel{\varphi}{\rightarrow}[e]$ the map $\varphi^{*}: R \mathbb{A}^{e} \rightarrow R \mathbb{A}^{d}$ is defined by $\varphi^{*}\left(T_{j}\right)=\sum_{\varphi(i)=j} T_{i}$. The resulting simplicial ring $R \mathbb{A}^{\cdot}: d \mapsto R \mathbb{A}^{d}$ has the element $T=T_{0}$ in degree 1 , which satisfies $\partial_{0}^{*} T=0$ and $\partial_{1}^{*} T=1$. Thus $T$ is an edge which connects 1 to 0 , making $R \mathbb{A}^{\cdot}$ a connected simplicial ring.

As for any connected simplicial ring, $R \mathbb{A}^{*}$ is actually contractible, for one can construct a homotopy $f: \Delta^{1} \times R \mathbb{A}^{\cdot} \rightarrow R \mathbb{A}^{\text {from }} 0$ to 1 by defining, for each $\ell \geq 0$, the map $f_{\ell}: \Delta_{\ell}^{1} \times R \mathbb{A}^{\ell} \rightarrow R \mathbb{A}^{\ell}$ with the formula $f_{\ell}(\varphi, r)=\left(\varphi^{*} T\right) \cdot r$. Any connected simplicial ring contains an element $T$ in degree 1 with $\partial_{0}^{*} T=0$ and $\partial_{1}^{*} T=1$.

If $F$ is a functor from rings to spaces, then we may consider the simplicial space $\left(d \mapsto F\left(R \mathbb{A}^{d}\right)\right.$; we let $F\left(R \mathbb{A}^{*}\right)$ denote it when there is no possibility of confusion. We see that $\pi_{0}\left|F\left(R \mathbb{A}^{\prime}\right)\right|$ is the quotient of $\pi_{0}|F(R)|$ by the equivalence relation generated by the requirement that $x(0) \sim x(1)$ for every $x=x(T) \in \pi_{0}|F(R[T])|=\pi_{0}\left|F\left(R \mathbb{A}^{1}\right)\right|$. This equivalence relation may be called polynomial homotopy, and the functor $F^{\prime}$ defined by $F^{\prime}(R)=\left|F\left(R \mathbb{A}^{\prime}\right)\right|$ may be said to be obtained from $F$ by working modulo polynomial homotopy.

There is a natural map $F \rightarrow F^{\prime}$ arising from the structure map $\mathbb{A}^{d} \rightarrow \operatorname{Spec}(\mathbb{Z})$. From $[20,1.7 \mathrm{i}$ and 2.4$]$ it follows that the natural map $F^{\prime} \rightarrow F^{\prime \prime}$ is a homotopy equivalence; this justifies the terminology. Another proof can be obtained by using the diagonal map $\mathbb{A}^{d} \rightarrow \mathbb{A}^{d} \times \mathbb{A}^{d}$ to define a map $\left|d \mapsto F\left(R \mathbb{A}^{d} \otimes_{R} R \mathbb{A}^{d}\right)\right| \rightarrow\left|d \mapsto F\left(R \mathbb{A}^{d}\right)\right|$ to serve as a homotopy inverse; one composite is the identity, and the other is simplicially homotopic to the identity via the evident affine homotopy.

For future use, we mention now that if we are given a simplicial exact category $d \mapsto \mathcal{P}_{d}$ then we define its $K$-theory space $K(\mathcal{P})$ to be $\left|d \mapsto K\left(\mathcal{P}_{d}\right)\right|$.

We are ready for the proposed filtration of Goodwillie and Lichtenbaum. We let $V^{t}=K\left(R \mathbb{A}^{\prime}, \mathbb{G}_{m}^{\wedge t}\right)=\left|d \mapsto K\left(R \mathbb{A}^{d}, \mathbb{G}_{m}{ }^{\wedge t}\right)\right|$, and define $W^{t}=\Omega^{-t} V^{t}$, making use of the deloopings available to spaces arising from $K$-theory.

We assume henceforth that $R$ is a regular noetherian ring. The Fundamental Theorem of $K$-theory then says that the map $K(R) \stackrel{\sim}{\longrightarrow} K(R[T])$ is a homotopy equivalence. It follows easily that the map

$$
K(R) \stackrel{\sim}{\longrightarrow} K\left(R \mathbb{A}^{\cdot}\right)
$$

is a homotopy equivalence. We see that $W^{0}=K\left(R \mathbb{A}^{*}, \mathbb{G}_{m}{ }^{\wedge 0}\right)=K\left(R \mathbb{A}^{*}, 1\right)=K\left(R \mathbb{A}^{*}\right) \sim$ $K(R)$. Multiplication by $U \in K_{1}\left(\mathbb{G}_{m}\right)$ gives a map $W^{t+1} \rightarrow W^{t}$. Assembling these two facts gives us a filtration

$$
K(R) \sim W^{0} \leftarrow W^{1} \leftarrow W^{2} \leftarrow \ldots
$$


of the type desired.

It is conceivable, at least when $R$ is local and thus (2.1) holds, that $W^{t}$ captures all of the weight $\geq t$ part of $K$-theory. Even if $R$ is not local, it is conceivable that $\pi_{n} W^{t}$ captures all of the weight $\geq t$ part of $K_{n} R$ provided $n \geq t$.

We see that

$$
\pi_{1} W^{1}=\pi_{0} V^{1}=\frac{K_{0}\left(R, \mathbb{G}_{m}\right)}{\operatorname{im~} K_{0}(R)+\operatorname{im} K_{0}\left(R[T], \mathbb{G}_{m}\right)},
$$

and it is an easy exercise to identify the latter group with $K_{1} R$ when $R$ is a regular noetherian ring. The main point of the exercise is that an elementary matrix $\left(\begin{array}{ll}1 & \alpha \\ 0 & 1\end{array}\right)$ can be deformed to the identity matrix by using the matrix $\left(\begin{array}{cc}1 & T \alpha \\ 0 & 1\end{array}\right)$ and letting $T$ go from 1 to 0.

Since $\pi_{1} W^{2}=\pi_{0} W^{2}=0$ we see that $\pi_{1} W^{1} / W^{2}=\pi_{1} W^{1}=K_{1}(R)$. The group $K_{1}$ has no weight zero part, and so when (2.1) holds, this group has pure weight 1 , but this fact is too trivial to be viewed as evidence for the general conjecture that $W^{t} / W^{t+1}$ has the right weight.

The group $\pi_{2} W^{2}$ is given explicitly as the quotient

$$
G W_{2} R=\pi_{2} W^{2} \cong \frac{K_{0}\left(R, \mathbb{G}_{m}{ }^{2}\right)}{\operatorname{im~} K_{0}\left(R, \mathbb{G}_{m} \times 1\right)+\operatorname{im} K_{0}\left(R, 1 \times \mathbb{G}_{m}\right)+\operatorname{im} K_{0}\left(R[T], \mathbb{G}_{m}{ }^{2}\right)}
$$

This formula amounts to an explicit definition of $G W_{2} R$ by generators and relations. Goodwillie has provided evidence for the correctness of the proposed filtration, for by a hard calculation he has shown that $G W_{2} R$ is isomorphic to $K_{2} R$ when $R$ is an algebraically closed field, and that $K_{2} R$ is a direct summand of $G W_{2} R$ when $R$ is a field which is either perfect or not of characteristic 2 . He expects to be able to show that the complementary summand vanishes.

An additional suggestion I received from Lichtenbaum is that the quotient $W^{t} / W^{t+1}$ might be

$$
\Omega^{-t}\left|d \mapsto K_{0}\left(R \mathbb{A}^{d}, \mathbb{G}_{m}^{\wedge t}\right)\right| .
$$

This is the explicit simplicial abelian group promised earlier, whose corresponding chain complex should figure in motivic cohomology. The techniques in this paper depend on the use of direct-sum $K$-theory (ignoring the exact sequences), so we end up with

$$
\Omega^{-t}\left|d \mapsto K_{0}^{\oplus}\left(R \mathbb{A}^{d}, \mathbb{G}_{m}^{\wedge t}\right)\right|
$$

instead. It is possible that (2.3) and (2.4) are homotopy equivalent, or equivalently, that the corresponding chain complexes are quasi-isomorphic; if this were not the case, it would be discouraging, for there are exact sequences arising from exterior powers which are not naturally split, and presumably we will need to use them to show that the operations arising from exterior powers behave properly here. On the other hand, it shouldn't be taken as a bad sign that these initial results involve direct sum $K$-theory, as the same was true for two of Quillen's three definitions of higher $K$-theory of a ring, and the definition most removed from consideration of exact sequences (the plus construction) was the one where the operations arising from exterior powers were first constructed. Moreover, direct sum $K$-theory might turn out to have certain computational advantages due to the simplicity of the construction. 


\section{Loop spaces for simplicial sets.}

We present now the definition of $\omega Y$ from [3] for a simplicial set $Y$ with base point $y_{0} \in Y_{0}$. It provides a combinatorial approximation to the loop space $\Omega|Y|$ in the sense that there is always a natural map $|\omega Y| \rightarrow \Omega|Y|$, and in favorable cases this map turns out to be a homotopy equivalence.

Given $A, B \in$ Ord we let $A B \in$ Ord denote the ordered set obtained by concatenating $A$ and $B$, with the elements of $A$ smaller than the elements of $B$. The natural inclusion maps $A \rightarrow A B \leftarrow B$ provide natural face maps $Y(A) \leftarrow Y(A B) \rightarrow Y(B)$. Let $P Y$ be the simplicial path space of edges in $Y$ with initial endpoint at $y_{0}$; it can be defined for $A \in$ Ord by

$$
(P Y)(A)=\lim _{\longleftarrow}\left(\begin{array}{cc} 
& Y([0] A) \\
& \downarrow \\
\left\{y_{0}\right\} \longrightarrow & Y([0])
\end{array}\right) .
$$

The space $|P Y|$ is contractible; one sees this with an explicit homotopy $h: \Delta^{1} \times P Y \rightarrow$ $P Y$ from $y_{0}$ to $1_{P Y}$ defined as follows. For $(\varphi, y) \in \Delta^{1}(A) \times(P Y)(A)$ we define $h(\varphi, y)=$ $t_{\varphi}^{*}(y)$ where $t_{\varphi}:[0] A \rightarrow[0] A$ is defined by

$$
\begin{aligned}
& t_{\varphi}(0)=0 \\
& t_{\varphi}(a)= \begin{cases}0 & \varphi(a)=0 \\
a & \varphi(a)=1\end{cases}
\end{aligned}
$$

The face maps $Y([0] A) \rightarrow Y(A)$ provide a projection map $P Y \rightarrow Y$. We define

$$
\omega Y=\lim _{\longleftarrow}\left(\begin{array}{cc} 
& P Y \\
& \downarrow \\
P Y \longrightarrow & Y
\end{array}\right) .
$$

The commutative square

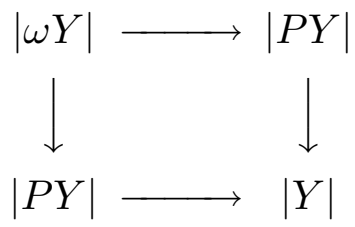

together with the contractibility of the space $|P Y|$ provides a natural map $|\omega Y| \rightarrow \Omega|Y|$.

A vertex of $\omega Y$ is nothing more than a pair of edges

$$
y_{0} \rightarrow y_{1} \leftarrow y_{0}
$$

in $Y$, and an edge of $\omega Y$ is a pair of triangles

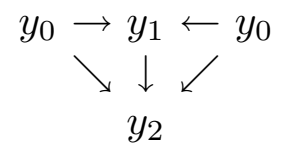

in $Y$. In these diagrams $y_{0}$ is the base point, and $y_{1}$ and $y_{2}$ are variable. A vertex of $\omega Y$ yields a loop in $|Y|$ which starts at $y_{0}$, follows one edge to $y_{1}$, and returns along the 
other edge to $y_{0}$, and an edge of $\omega Y$ yields a homotopy between two such loops. This motivates the definition of $\omega Y$.

In the case where $Y$ is actually a category (regarded as a simplicial set by identifying it with its nerve) the simplicial set $P Y$ is the same as the comma category $y_{0} / Y$, and $\omega Y$ is the comma category $\left(y_{0}, y_{0}\right) / \Delta_{Y}$, where $\Delta_{Y}: Y \rightarrow Y \times Y$ is the diagonal map. The triangles in the diagram above are then to be interpreted as commutative triangles. One can also write $\omega Y$ as the iterated comma category $y_{0} / F$, where $F$ is the projection $y_{0} / Y \stackrel{F}{\longrightarrow} Y$; we'll use this later.

We remark that if $Y$ is a simplicial group, then the map $|\omega Y| \rightarrow \Omega|Y|$ is always a homotopy equivalence. To prove this one assumes that $Y$ is connected and uses the Kan extension property to show that $P Y \rightarrow Y$ is a surjective map of simplicial groups, hence is a Kan fibration.

In [3] it was shown that $|\omega S \mathcal{M}| \rightarrow \Omega|S \mathcal{M}|$ is a homotopy equivalence, where $\mathcal{M}$ is an exact category, and $S$ is the $S$-construction of Waldhausen; we defined $G . \mathcal{M}=\omega S \mathcal{M}$. (See also [7] and [10].)

It is worth observing that $\left|\omega S^{\oplus} \mathcal{M}\right| \rightarrow \Omega\left|S^{\oplus} \mathcal{M}\right|$ is a homotopy equivalence, where $\left|S^{\oplus} \mathcal{M}\right|$ is the direct-sum $K$-theory defined in section 4 below; the proof can be obtained by applying lemma 2.1 of [3]. Indeed, one of Quillen's original constructions for $K$ theory (described in [4]) was the category $S^{-1} S \mathcal{M}$ introduced below, which is essentially equivalent to $\omega S^{\oplus} \mathcal{M}$. He introduced $S^{-1} S \mathcal{M}$ for the purpose of relating the $K$-theory of $\mathcal{P}(R)$, as defined with the $Q$-construction, to the $K$-theory defined via the infinite general linear group of $R$. Quillen has also remarked that looping a space combinatorially seems to be a good way to perform computations, but delooping seems to be a good way to prove general theorems. As an example of the former, one could cite the fact that Milnor's $K_{2}$ group agrees with Quillen's - to prove this, one seems to need a $K$-theory space where $K_{2}$ appears as $\pi_{2}$, such as $B G \ell(R)^{+}$. As an example of the latter, one could cite the localization theorem for abelian categories of Quillen - to prove it, one seems to need a $K$-theory space where $K_{2}$ appears as $\pi_{3}$, such as the the $Q$-construction of Quillen. In line with this observation, in this paper we are forming multiple loop spaces, with the hope that they will lead to specific computations.

\section{Direct sum $K$-theory.}

An exact category in which every exact sequence splits is completely determined by the underlying additive category. It is well known from work of Quillen and Waldhausen that the $K$-theory of such a category can be constructed in various ways. We begin by describing a slight modification of theire ideas.

Let $S^{\oplus} \mathcal{M}$ denote the version of the $S$-construction where the short exact sequences are replaced by direct sum diagrams. We make this precise as follows. For $A \in$ Ord we define a category $\operatorname{Sub}(A)$ whose objects are the pairs $(i, j)$ with $i \leq j \in A$, and where there is an (unique) arrow $\left(i^{\prime}, j^{\prime}\right) \rightarrow(i, j)$ exactly when $i^{\prime} \leq i \leq j \leq j^{\prime}$. We say that a functor $M: \operatorname{Sub}(A) \rightarrow \mathcal{M}$ is additive if $M(i, i)=0$ for all $i \in A$, and for all $i \leq j \leq k \in A$ the map $M(i, k) \rightarrow M(i, j) \oplus M(j, k)$ is an isomorphism. Here 0 denotes a previously chosen zero object of $\mathcal{M}$. The set of such exact functors is denoted by $\operatorname{Add}(\operatorname{Sub}(A), \mathcal{M})$.

Definition 4.1. We define the simplicial set $S^{\oplus} \mathcal{M}$ by setting

$$
\left(S^{\oplus} \mathcal{M}\right)(A)=\operatorname{Add}(\operatorname{Sub}(A), \mathcal{M}) .
$$


The simplicial set $S^{\oplus} \mathcal{M}$ is closely related to $N_{\Gamma} \mathcal{M}$ of Waldhausen [17, p. 174], but doesn't include as many direct sum diagrams. An $n$-simplex $M \in S_{n}^{\oplus} \mathcal{M}$ may be thought of as a compatible collection of direct sum diagrams $M(i, j) \cong M(i, i+1) \oplus \cdots \oplus M(j-$ $1, j)$. There is a natural map $S^{\oplus} \mathcal{M} \rightarrow S \mathcal{M}$ which converts each direct sum diagram $M(i, k) \cong M(i, j) \oplus M(j, k)$ into the short exact sequence $0 \rightarrow M(i, j) \rightarrow M(i, k) \rightarrow$ $M(j, k) \rightarrow 0$.

Definition 4.2. If $\mathcal{M}$ is a simplicial additive category, then we define $S^{\oplus} \mathcal{M}=(d \mapsto$ $\left.S^{\oplus} \mathcal{M}_{d}\right)$. When $\mathcal{M}$ is an additive category or a simplicial additive category, we define $K^{\oplus} \mathcal{M}=\Omega\left|S^{\oplus} \mathcal{M}\right|$, and refer to its homotopy groups as the direct sum $K$-groups, $K_{i}^{\oplus} \mathcal{M}$.

For rings $R$ and $S$ we define $K^{\oplus}(R, S)=K^{\oplus} \mathcal{P}(R, S)$.

Now we extend the definition of $S^{\oplus}$ to cubes of exact categories just as the definition of $S$ was extended to cubes in $[8, \S 4]$, yielding multirelative direct-sum $K$-theory. Much of this material is essentially the same as the treatment of $N_{\Gamma}(W, V)$ in $[17$, p. 174]. Let $L$ be a symbol, and consider $\{L\}$ to be an ordered set. Given an $n$-dimensional cube of additive categories $\mathcal{M}$, we define an $n$-fold multisimplicial additive category $C^{\oplus} \mathcal{M}$ as a functor from $\left(\mathrm{Ord}^{n}\right)^{\text {op }}$ to the category of exact categories by letting $C^{\oplus} \mathcal{M}\left(A_{1}, \ldots, A_{n}\right)$ be the set

$$
\operatorname{Add}\left(\left[\operatorname{Sub}\left(A_{1}\right) \rightarrow \operatorname{Sub}\left(\{L\} A_{1}\right)\right] \otimes \cdots \otimes\left[\operatorname{Sub}\left(A_{n}\right) \rightarrow \operatorname{Sub}\left(\{L\} A_{n}\right)\right], \mathcal{M}\right)
$$

of multi-additive natural transformations. When $n=0$, we may identify $C^{\oplus} \mathcal{M}$ with $\mathcal{M}$. We define $S^{\oplus} \mathcal{M}$ to be $S^{\oplus} C^{\oplus} \mathcal{M}$, the result of applying the $S^{\oplus}$ construction of Waldhausen degreewise. It is an $n+1$-fold multisimplicial set.

Lemma 4.3. Suppose we are given an additive map $\mathcal{M}^{\prime} \rightarrow \mathcal{M}$ of $n$-dimensional cubes of additive categories. Let $\left[\mathcal{M}^{\prime} \rightarrow \mathcal{M}\right]$ denote the corresponding $n+1$-dimensional cube of additive categories.

(a) There is a fibration sequence

$$
S^{\oplus}[0 \rightarrow \mathcal{M}] \rightarrow S^{\oplus}\left[\mathcal{M}^{\prime} \rightarrow \mathcal{M}\right] \rightarrow S^{\oplus}\left[\mathcal{M}^{\prime} \rightarrow 0\right]
$$

(b) The space $S^{\oplus}[\mathcal{M} \stackrel{1}{\rightarrow} \mathcal{M}]$ is contractible.

(c) $S^{\oplus}[0 \rightarrow \mathcal{M}]$ is homotopy equivalent to $S^{\oplus} \mathcal{M}$.

(d) $S^{\oplus}[\mathcal{M} \rightarrow 0]$ is a delooping of $S^{\oplus} \mathcal{M}$.

(e) There is a fibration sequence $S^{\oplus} \mathcal{M}^{\prime} \rightarrow S^{\oplus} \mathcal{M} \rightarrow S^{\oplus}\left[\mathcal{M}^{\prime} \rightarrow \mathcal{M}\right]$.

Proof. One uses the additivity theorem of Waldhausen, just as in [18, p. 343] or [17, p. 182-184]. Actually, since these are direct sum diagrams instead of short exact sequences, the analogue of the additivity theorem we actually use is much easier.

Here is the precise definition of Quillen's category $S^{-1} S \mathcal{M}$, for an additive category $\mathcal{M}$. We define a split monomorphism $i: M \stackrel{\leftarrow}{\longmapsto} N$ in $\mathcal{M}$ to be a pair consisting of an admissible monomorphism $i: M \longmapsto N$ and an admissible epimorphism $p: N \rightarrow M$ such that $p i=1$. There is an evident notion of composition for split monomorphisms. An object of $S^{-1} S \mathcal{M}$ is a pair $\left(M^{\prime}, M^{\prime \prime}\right)$ of objects of $\mathcal{M}$, and an arrow $\left(M^{\prime}, M^{\prime \prime}\right) \rightarrow$ $\left(N^{\prime}, N^{\prime \prime}\right)$ of $S^{-1} S \mathcal{M}$ is a triple $\left(f^{\prime}, f^{\prime \prime}, h\right)$ consisting of split monomorphisms $f^{\prime}: M^{\prime} \stackrel{\sharp}{\longmapsto} N^{\prime}$ 
and $f^{\prime \prime}: M^{\prime \prime} \stackrel{\leftarrow}{\longmapsto} N^{\prime \prime}$, with an isomorphism $h: \operatorname{ckr} f^{\prime} \stackrel{\cong}{\longrightarrow} \operatorname{ckr} f^{\prime \prime}$. (It is possible to specify the isomorphism $h$ without having picked objects to serve as cokernels of $f^{\prime}$ and $f^{\prime \prime}$, for we may specify such an $h$ simultaneously for all possible choices of cokernels. Alternatively, we could specify $h$ by means of the map $\left(\begin{array}{ll}0 & 0 \\ 0 & h\end{array}\right): N^{\prime} \rightarrow N^{\prime \prime}$.)

In the case where $M^{\prime}=0$ and $M^{\prime \prime}=0$ then $f^{\prime}$ and $f^{\prime \prime}$ are uniquely determined and can be omitted from the notation; thus an arrow $(0,0) \stackrel{h}{\longrightarrow}\left(N^{\prime}, N^{\prime \prime}\right)$ is the same thing as an isomorphism $h: N^{\prime} \cong N^{\prime \prime}$. In the case where $f^{\prime}$ and $f^{\prime \prime}$ are isomorphisms, then the isomorphism $h$ is uniquely determined and can be omitted from the notation; thus an isomorphism $\left(M^{\prime}, M^{\prime \prime}\right) \stackrel{\left(f^{\prime}, f^{\prime \prime}\right)}{\longrightarrow}\left(N^{\prime}, N^{\prime \prime}\right)$ is simply a pair of isomorphisms. Any arrow of $S^{-1} S \mathcal{M}$ is isomorphic to one of the form

$$
\left(f^{\prime}, f^{\prime \prime}, 1_{X}\right):\left(M^{\prime}, M^{\prime \prime}\right) \rightarrow\left(M^{\prime} \oplus X, M^{\prime \prime} \oplus X\right)
$$

where $f^{\prime}$ and $f^{\prime \prime}$ are the split inclusions onto the first factor.

Composition of arrows in the category $S^{-1} S \mathcal{M}$ is done by composing the split monomorphisms and taking the direct sum of the isomorphisms. This can be made a bit more explicit: we leave that as an exercise. The object $(0,0)$ will be the base point in $S^{-1} S \mathcal{M}$.

There is an evident map $\omega S^{\oplus} \mathcal{M} \rightarrow S^{-1} S \mathcal{M}$. A map the other way can be obtained by first choosing cokernels for all admissible monomorphisms in $\mathcal{M}$, so using the techniques involved in Lemma 3.1.1 of [3] and the paragraph following it, one sees that the two maps are inverse homotopy equivalences. Here is another approach to the equivalence which roughly follows Waldhausen's reasoning in part (2) of the Corollary on p. 335 of [18]. For $A \in$ Ord let

$$
\Gamma(A)=\lim \left(\begin{array}{c}
A \\
\downarrow \\
{[0] A}
\end{array}\right),
$$

and interpret $\left(\omega S^{\oplus} \mathcal{M}\right)(A)$ as the set of additive functors $\operatorname{Sub}(\Gamma(A)) \rightarrow \mathcal{M}$. Let $0^{\prime}$ and $0^{\prime \prime}$ denote the two copies of 0 in $\Gamma(A)$. Let $I s^{\prime} \omega S_{k}^{\oplus} \mathcal{M}$ denote the groupoid of those natural isomorphisms $M \rightarrow N$ between elements of $\omega S_{k}^{\oplus} \mathcal{M}$ that induce identity arrows $M\left(0^{\prime}, i\right) \rightarrow N\left(0^{\prime}, i\right)$ and $M\left(0^{\prime \prime}, i\right) \rightarrow N\left(0^{\prime \prime}, i\right)$ for each $i \in[k]$. We see that $S^{-1} S_{k} \mathcal{M}$ is the set $\pi_{0} I s^{\prime} \omega S_{k}^{\oplus} \mathcal{M}$ of isomorphism classes in the groupoid, and since the groupoid's objects have trivial automorphism groups, the map $I s^{\prime} \omega S_{k}^{\oplus} \mathcal{M} \rightarrow S^{-1} S_{k} \mathcal{M}$ is a homotopy equivalence. Let $I s^{\prime} \omega S^{\oplus} \mathcal{M}$ denote the bisimplicial set $\left(k \mapsto I s^{\prime} \omega S_{k}^{\oplus} \mathcal{M}\right)$. The map $I s^{\prime} \omega S^{\oplus} \mathcal{M} \rightarrow S^{-1} S \mathcal{M}$ is a homotopy equivalence, because it is a homotopy equivalence in each degree. The degeneracy map $\omega S^{\oplus} \mathcal{M}=I s_{0}^{\prime} \omega S^{\oplus} \mathcal{M} \rightarrow I s_{k}^{\prime} \omega S^{\oplus} \mathcal{M}$ is a simplicial homotopy equivalence: the inverse is the face map arising from the inclusing $[0] \rightarrow[k]$ sending 0 to $k$, and the simplicial homotopy required can be constructed explicitly by a formula essentially the same as that of Waldhausen displayed in the proof of Lemma 3.1.1 of [3].

Quillen proved in [4] that $K(\mathcal{M}) \sim\left|S^{-1} S \mathcal{M}\right|$ when $\mathcal{M}$ is an exact category in which every exact sequence splits. The isomorphism $\pi_{0} S^{-1} S \mathcal{M} \rightarrow K_{0} \mathcal{M}$ is implemented by sending the component containing $\left(P^{\prime}, P^{\prime \prime}\right)$ to the class $\left[P^{\prime}\right]-\left[P^{\prime \prime}\right]$ in $K_{0}(\mathcal{M})$. 
Let's examine the category $\omega S^{-1} S \mathcal{M}$ more closely now. The objects are diagrams

$$
(0,0) \stackrel{\alpha}{\longrightarrow}\left(P^{\prime}, P^{\prime \prime}\right) \stackrel{\beta}{\longleftarrow}(0,0)
$$

in $S^{-1} S \mathcal{M}$, and the arrows are commutative diagrams

$$
\begin{aligned}
(0,0) \stackrel{\alpha}{\longrightarrow}\left(P^{\prime}, P^{\prime \prime}\right) \stackrel{\beta}{\longleftarrow}(0,0) \\
\qquad \underset{\left(Q^{\prime}, Q^{\prime \prime}\right)}{\swarrow}
\end{aligned}
$$

By taking the vertical arrow in this diagram to be the isomorphism

$$
\left(\alpha, 1_{P^{\prime \prime}}\right):\left(P^{\prime}, P^{\prime \prime}\right) \rightarrow\left(P^{\prime \prime}, P^{\prime \prime}\right)
$$

we see that any object of $\omega S^{-1} S \mathcal{M}$ is isomorphic to an object of the form

$$
(0,0) \stackrel{1_{P}}{\longrightarrow}(P, P) \stackrel{\beta}{\longleftarrow}(0,0)
$$

in which the left hand arrow arises from the identity map of $P$, and the right hand arrow is determined by an automorphism $\beta \in$ Aut $P$. The full subcategory $\omega^{\prime} S^{-1} S \mathcal{M}$ of $\omega S^{-1} S \mathcal{M}$ consisting of such objects is equivalent to $\omega S^{-1} S \mathcal{M}$. An arrow in $\omega^{\prime} S^{-1} S \mathcal{M}$ is a commutative diagram

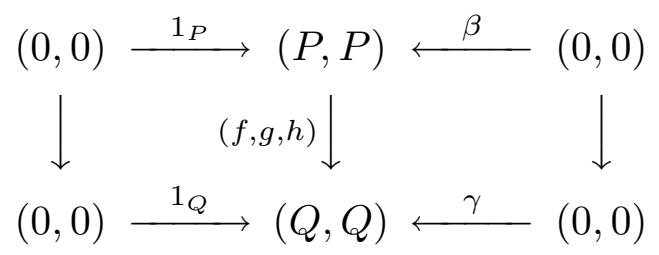

The commutativity of the left hand square implies that the maps $1_{Q}, 1_{P}$, and $h$ provide an isomorphism from the direct sum diagram $Q \cong P \oplus \operatorname{ckr} f$ determined by $f$ to the direct sum diagram $Q \cong P \oplus \operatorname{ckr} g$ determined by $g$. This implies that $f=g$ and $h=1$, so if we write $X=\operatorname{ckr} f$, and write $Q$ as $P \oplus X$, then we see that $\gamma=\beta \oplus 1_{X}$.

We can rephrase the result of the discussion in the previous paragraph as follows. The category $\omega S^{-1} S \mathcal{M}$ is equivalent to the category $\mathcal{C}$ where an object is any pair $(P, \beta)$ with $P \in \mathcal{M}$ and $\beta \in$ Aut $P$, and an arrow $(P, \beta) \rightarrow(Q, \gamma)$ is any split monomorphism $P \stackrel{\leftrightarrow}{\leftrightarrows} Q$ with respect to which one has the equation $\gamma=\beta \oplus 1$. The objects of $\mathcal{C}$ are themselves the objects of the exact category $\mathcal{M}\left(\mathbb{G}_{m}\right)$, the arrows of $\mathcal{C}$ are also the objects of an exact category, and indeed, the nerve of $\mathcal{C}$ can be interpreted as a simplicial exact category. (If I had enough nerve, I would simply inform you that $\mathcal{C}$ is the category of objects of a category exact category, and leave it at that!) We will let $\mathcal{C}$ also denote this simplicial additive category. There is a map $C^{\oplus} \mathcal{M}\left(\mathbb{G}_{m}{ }^{\wedge 1}\right) \rightarrow \mathcal{C}$ which amounts to forgetting some choices of cokernels, so as before, the map is a homotopy equivalence. It is also true that $S^{\oplus} C^{\oplus} \mathcal{M}\left(\mathbb{G}_{m}{ }^{\wedge 1}\right) \rightarrow S^{\oplus} \mathcal{C}$ is a homotopy equivalence. Thus we have a homotopy equivalence

$$
K^{\oplus} \mathcal{M}\left(\mathbb{G}_{m}^{\wedge 1}\right) \sim K^{\oplus} \omega S^{-1} S \mathcal{M}
$$


Now we give an example to show why the map

$$
\left|\omega S^{-1} S \mathcal{M}\right| \rightarrow \Omega\left|S^{-1} S \mathcal{M}\right|
$$

is not always a homotopy equivalence. Let $R$ be a ring. We take for $\mathcal{M}$ the exact category $\mathcal{F}(R)$ finitely generated free $R$-modules. (Taking $\mathcal{M}=\mathcal{P}(R)$ works just as well.) To see that the map

$$
\left|\omega S^{-1} S \mathcal{F}(R)\right| \rightarrow \Omega\left|S^{-1} S \mathcal{F}(R)\right|
$$

is not a homotopy equivalence it is sufficient to show that the induced map

$$
\pi_{0}\left|\omega S^{-1} S \mathcal{F}(R)\right| \rightarrow K_{1}(R)
$$

is not an isomorphism. It follows from the discussion above that the set on the left is $\pi_{0}|\mathcal{C}|=\pi_{0}\left|\operatorname{obj} \mathcal{F}(R)\left(\mathbb{G}_{m}^{\wedge 1}\right)\right|$, and so it is the quotient of the set

$$
\coprod_{n=1}^{\infty} G \ell_{n}(R)
$$

by the equivalence relation generated by the following two requirements.

$$
\begin{aligned}
& A \sim C A C^{-1} \\
& A \sim A \oplus 1_{k}
\end{aligned}
$$

Here $A$ and $C$ are in $G \ell_{n}(R)$ for some $n$, and $1_{k}$ is the $k$ by $k$ identity matrix. It follows that $\pi_{0}\left|\omega S^{-1} S \mathcal{F}(R)\right|$ is the set of conjugacy classes in $G \ell(R)$, and this is definitely not the same thing as $K_{1}(R)$, as it isn't even a group under $\oplus$.

Even though we know that $\left|\omega S^{-1} S \mathcal{M}\right|$ is not the loop space of $\left|S^{-1} S \mathcal{M}\right|$, it is worth considering, in the remaining paragraphs of this section, how close one comes in trying to prove it, for later on we will eliminate the obstacle by replacing $\mathcal{M}$ by a simplicial additive category over a connected simplicial ring.

For a pointed simplicial set, space, or category $Y$ we let $I_{*} Y$ denote the connected component of $Y$ containing the base point. (If $y$ is a point of $Y$, we let $I_{y} Y$ denote the connected component of $Y$ containing $y$.)

The loops used in the construction of $\omega S^{-1} S \mathcal{M}$ start at the base point $*=(0,0)$, and don't wander into other components, so $\omega S^{-1} S \mathcal{M}=\omega I_{*} S^{-1} S \mathcal{M}$. In order to prove that the square

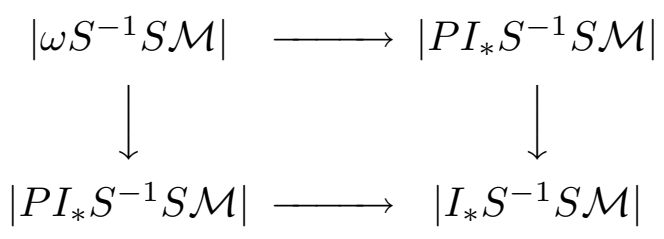

is homotopy cartesian, we could try to compute the homotopy fiber of the map $F$ : $P I_{*} S^{-1} S \mathcal{M} \rightarrow I_{*} S^{-1} S \mathcal{M}$ using Theorem B of Quillen [15]. (The fiber $(0,0) / F$ is 
evidently the same as $\omega S^{-1} S \mathcal{M}$.) In order to apply Theorem B, we would need to show that for any arrow $\left(P^{\prime}, P^{\prime \prime}\right) \rightarrow\left(V^{\prime}, V^{\prime \prime}\right)$ of $I_{*} S^{-1} S \mathcal{M}$ the transition map

$$
\left(V^{\prime}, V^{\prime \prime}\right) / F \rightarrow\left(P^{\prime}, P^{\prime \prime}\right) / F
$$

on right fibers is a homotopy equivalence. A necessary condition for this to succeed is that each fiber $\left(P^{\prime}, P^{\prime \prime}\right) / F$ be nonempty, for at least one of the fibers is nonempty, and the base space $I_{*} S^{-1} S \mathcal{M}$ is connected. The category $\left(P^{\prime}, P^{\prime \prime}\right) / F$ is nonempty if and only if there is a diagram of the form

$$
\left(P^{\prime}, P^{\prime \prime}\right) \rightarrow\left(Q^{\prime}, Q^{\prime \prime}\right) \leftarrow(0,0)
$$

in $X_{0}$ which connects $\left(P^{\prime}, P^{\prime \prime}\right)$ to the base point, for such diagrams are the objects of $\left(P^{\prime}, P^{\prime \prime}\right) / F$. In fact, there always is such a diagram when $\left(P^{\prime}, P^{\prime \prime}\right) \in I_{*} S^{-1} S \mathcal{M}$, for then $\left[P^{\prime}\right]-\left[P^{\prime \prime}\right]=0$ in $K_{0}^{\oplus} \mathcal{M}$, and it follows that $P^{\prime}$ and $P^{\prime \prime}$ are stably isomorphic. A stable isomorphism $\theta: P^{\prime} \oplus Q \cong P^{\prime \prime} \oplus Q$ yields a diagram

$$
\left(P^{\prime}, P^{\prime \prime}\right) \rightarrow\left(P^{\prime} \oplus Q, P^{\prime \prime} \oplus Q\right) \stackrel{\theta}{\longleftarrow}(0,0)
$$

of the desired type.

Since every object of $\left(P^{\prime}, P^{\prime \prime}\right) / F$ is isomorphic to one of this form, we will call $\left(P^{\prime}, P^{\prime \prime}\right) / F$ the category of stable isomorphisms from $P^{\prime}$ to $P^{\prime \prime}$, and let $\operatorname{Stab}\left(P^{\prime}, P^{\prime \prime}\right)$ denote it. A worthwhile exercise is to describe the arrows of this category in explicit terms.

One might consider using the $G$-construction of [3] instead of $S^{-1} S$ to try to bring the exact sequences of $\mathcal{M}$ into play, but the right fibers would not all be nonempty. For a simple example, take for $\mathcal{M}$ the category of finitely generated abelian groups, and consider the object $(\mathbb{Z} / 2 \mathbb{Z}, 0) \in I_{*} G \mathcal{M}$. Connecting it to the origin by a path

$$
(\mathbb{Z} / 2,0) \rightarrow(M, M) \stackrel{1_{M}}{\longleftarrow}(0,0)
$$

amounts to finding a pair of exact sequences

$$
\begin{array}{r}
0 \rightarrow \mathbb{Z} / 2 \rightarrow M \rightarrow N \rightarrow 0 \\
0 \rightarrow 0 \rightarrow M \rightarrow N \rightarrow 0
\end{array}
$$

in $\mathcal{M}$, and this can't be done, because any surjective endomorphism of the noetherian module $M$ is an isomorphism. Such a simple example doesn't seem to exist in the opposite category, though, so this offers some hope of handling the $G$-construction for certain sorts of exact categories.

It turns out that when $\mathcal{M}$ is the category of modules of finite length over a Dedekind domain $R$, the right fibers are all nonempty (still using the $G$-construction). To see this, let $P$ and $Q$ be two such modules with the same simple factors, and assume, without loss of generality, that they are primary for the prime $p$. Write $P \cong \bigoplus_{i=1}^{n} R / p^{a_{i}}$ and $Q \cong \bigoplus_{i=1}^{n} R / p^{b_{i}}$. For $1 \leq k \leq n$ define $c_{k}=\sum_{i=1}^{k}\left(a_{i}-b_{i}\right)+K$, where $K$ is an integer sufficiently large to ensure that $c_{k} \geq a_{k}$ for all $k$. One checks that $c_{k}-a_{k}=c_{k-1}-b_{k}$ for $1<k \leq n$ and $c_{1}-a_{1}=c_{n}-b_{1}$. It follows that there are exact sequences

$$
\begin{aligned}
& 0 \rightarrow P \rightarrow M \rightarrow N \rightarrow 0 \\
& 0 \rightarrow Q \rightarrow M \rightarrow N \rightarrow 0
\end{aligned}
$$

with $M=\bigoplus R / p^{c_{i}}$ and $N=\bigoplus_{k=1}^{n} R / p^{c_{k}-a_{k}} \cong \bigoplus_{k=2}^{n} R / p^{c_{k-1}-b_{k}} \oplus R / p^{c_{n}-b_{1}}$. 


\section{Fibers for maps of simplicial categories.}

Let $F: \mathcal{B} \rightarrow \mathcal{C}$ be a map of simplicial categories. Waldhausen [19, Section 4] provides us with a method of computing the homotopy fiber of $|F|$ in certain cases, which we proceed to explain. For any $\ell \geq 0$ and any object $C \in \mathcal{C}_{\ell}$ the right fiber $C / F$ is the simplicial category defined in terms of the comma categories for the component functors $F_{d}$ of $F$ as follows.

$$
(C / F)_{d}=\coprod_{\varphi:[d] \rightarrow[\ell]} \varphi^{*} C / F_{d}
$$

Notice that the index set for the coproduct depends on $d$, so $C / F$ is not a disjoint union of simplicial categories.

We write an object of the category $(C / F)_{d}$ as a triple $(\varphi, f, B)$ with $\varphi:[d] \rightarrow[\ell]$, $f: \varphi^{*} C \rightarrow F_{d} B$, and $B \in \mathcal{B}_{d}$. The face map $\delta^{*}:(C / F)_{d} \rightarrow(C / F)_{d^{\prime}}$ corresponding to a map $\delta:\left[d^{\prime}\right] \rightarrow[d]$ is defined by the formula $\delta^{*}(\varphi, f, B)=\left(\delta^{*} \varphi, \delta^{*} f, \delta^{*} B\right)$. Here $\delta^{*} \varphi=\varphi \circ \delta$ is the image of $\varphi$ under the face map $\delta^{*}: \Delta_{d}^{\ell} \rightarrow \Delta_{d^{\prime}}^{\ell}$.

There are transition maps of two types. Given an arrow $p: C^{\prime} \rightarrow C$ in $\mathcal{C}_{\ell}$ the transition map

$$
u(p): C / F \rightarrow C^{\prime} / F
$$

is defined by $u(p)(\varphi, f, B)=\left(\varphi, f \circ \varphi^{*} p, B\right)$. Given a map $\lambda:\left[\ell^{\prime}\right] \rightarrow[\ell]$ the transition map $v_{\lambda}:\left(\lambda^{*} C\right) / F \rightarrow C / F$ is defined by $v_{\lambda}(\varphi, f, B)=(\lambda \circ \varphi, f, B)$.

Waldhausen's Theorem $\mathrm{B}^{\prime}$ states [19] that if the transition maps are all homotopy equivalences, then for any $C$ the square

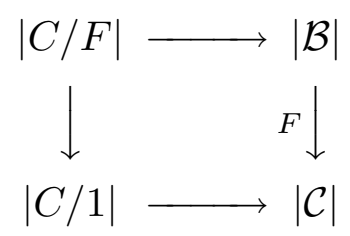

is a homotopy cartesian square, and the space $|C / 1|$, where 1 denotes the identity map on $\mathcal{C}$, is contractible.

In the case where $C$ is the base point object $C_{0}$ of $X_{0}$, then $\ell=0$, and for each $d \geq 0$ there is a unique map $\varphi_{d}:[d] \rightarrow[0]$. Thus the disjoint union in the definition of $\left(C_{0} / F\right)_{d}$ has just one term, and we find that $\left(C_{0} / F\right)_{d}=\varphi_{d}^{*} C / F_{d}$.

Theorem 5.3. With notation as above, if each category $\mathcal{C}_{\ell}$ is connected, and all transition maps arising from arrows $C^{\prime} \rightarrow C$ are homotopy equivalences, then the square (5.2) is homotopy cartesian.

Proof. Given a map $\beta:[m] \rightarrow[\ell]$, we may show that the transition map $\left(\beta^{*} C\right) / F \rightarrow$ $C / F$ is a homotopy equivalence as follows. Let $C_{0}$ be any object of $\mathcal{C}_{0}$, and using connectedness locate a path

$$
\varphi_{\ell}^{*} C_{0}=C_{0}^{\prime} \rightarrow C_{1}^{\prime} \leftarrow C_{2}^{\prime} \rightarrow \ldots \leftarrow C_{n}^{\prime}=C
$$

in $\mathcal{C}_{\ell}$ which connects $C$ to the base point. As Waldhausen remarks [19, Addendum, p. 166], it is easy to check that there is a homeomorphism $\left|\varphi_{\ell}^{*} C_{0} / F\right| \cong \Delta^{\ell} \times\left|C_{0} / F\right|$, in 
terms of which the transition map $\left|\varphi_{\ell}^{*} C_{0} / F\right| \rightarrow\left|C_{0} / F\right|$ associated to $\varphi_{\ell}$ is the projection on the second factor, and is thus a homotopy equivalence. The transition map for $\varphi_{m}$ is also a homotopy equivalence, and since $\varphi_{\ell} \beta=\varphi_{m}$, we see that the transition map $\left|\beta^{*} C_{0}^{\prime} / F\right| \rightarrow\left|C_{0}^{\prime} / F\right|$ associated to $\beta$ is a homotopy equivalence, too. Now we prove that $\left|\beta^{*} C_{n}^{\prime} / F\right| \rightarrow\left|C_{n}^{\prime} / F\right|$ is a homotopy equivalence by using a sequence of commutative squares

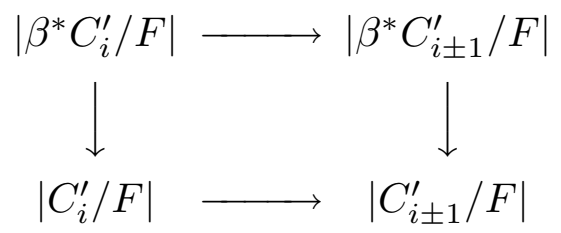

in which the horizontal maps are transition maps coming from arrows, thus are homotopy equivalences by assumption, and the vertical maps are transition maps for $\beta$, one of which is a homotopy equivalence by induction, thereby allowing us to conclude that the other vertical map is a homotopy equivalence.

\section{Homotopies between transition maps.}

In this section we develop a method for producing homotopies between transition maps like $u(p)$ above.

First we introduce some notation. If $X$ and $Y$ are simplicial sets, we let $X \otimes Y$ denote the bisimplicial set $(p, q) \mapsto X_{p} \times Y_{q}$. A symbol like $X \times Y \otimes Z$ will be interpreted to mean $(X \times Y) \otimes Z$, i.e., the operator $\times$ will be regarded as having higher precendence that $\otimes$. We let $\Delta^{\ell, m}$ denote $\Delta^{\ell} \otimes \Delta^{m}$, and $\Delta^{\ell \times m}$ will denote $\Delta^{\ell} \times \Delta^{m}$. We combine this notation and let $\Delta^{\ell \times m, n}$ denote $\Delta^{\ell} \times \Delta^{m} \otimes \Delta^{n}$.

Yoneda's lemma gives a correspondence $X_{p} \cong \operatorname{Hom}\left(\Delta^{p}, X\right)$, so if $\varphi \in \Delta_{m}^{p}$ and $f \in X_{p}$, we may write variously $\varphi^{*}(f)=f(\varphi)=f \circ \varphi$.

If $X$ is a bisimplicial set, then $X_{\ell, m}=\operatorname{Hom}\left(\Delta^{\ell, m}, X\right)$, so if $\varphi \in \Delta_{p}^{\ell}, \psi \in \Delta_{q}^{m}$, and $f \in X_{\ell, m}$, we may write variously $(\varphi \otimes \psi)^{*}(f)=f \circ(\varphi \otimes \psi)=f(\varphi, \psi)$. We define $X_{\ell \times m, n}=\operatorname{Hom}\left(\Delta^{\ell \times m, n}, X\right)$. Given $\varphi \in \Delta_{p}^{\ell}, \psi \in \Delta_{p}^{m}, \tau \in \Delta_{q}^{n}$, and $g \in X_{\ell \times m, n}$ we will write $((\varphi, \psi) \otimes \tau)^{*}(g)=g((\varphi, \psi), \tau) \in X_{p q}$. We will often use $g(\varphi, \psi)$ to denote $g((\varphi, \psi), 1) \in X_{p n}$.

Let $\mathcal{C}$ be a simplicial category, so that for $\ell \geq 0$ we have the category $\mathcal{C}_{\ell}$. Identifying a category with its nerve allows us to write

$$
\begin{aligned}
\mathcal{C}_{\ell 0} & =\operatorname{obj} \mathcal{C}_{\ell} \\
\mathcal{C}_{\ell 1} & =\operatorname{arr} \mathcal{C}_{\ell} \\
\operatorname{src} & =\partial_{1}^{*}: \mathcal{C}_{\ell 1} \rightarrow \mathcal{C}_{\ell 0} \\
\operatorname{tar} & =\partial_{0}^{*}: \mathcal{C}_{\ell 1} \rightarrow \mathcal{C}_{\ell 0}
\end{aligned}
$$

and to think of $\mathcal{C}$ as a bisimplicial set $(\ell, m) \mapsto \mathcal{C}_{\ell m}$. We let $\mathcal{C}_{* \times \ell, m}$ denote the simplicial set $d \mapsto \mathcal{C}_{d \times \ell, m}$, and we think of an element $g \in \mathcal{C}_{d \times \ell, m}$ as a $d$-parameter family of $(\ell, m)$-simplices of $\mathcal{C}$. We say that $g$ is constant if $g$ is totally degenerate, i.e., there exists $b \in \mathcal{C}_{0 \times \ell, m}=\mathcal{C}_{\ell, m}$ such that $g=\varphi_{d}^{*}(b)$. The subsimplicial set of $\mathcal{C}_{* \times \ell, m}$ generated by $b$ will be denoted by $\{b\}$. 
Given objects $C^{\prime}$ and $C$ of $\mathcal{C}_{\ell}$ we define $\underline{\operatorname{Hom}}_{\mathcal{C}}\left(C^{\prime}, C\right)$ to be the simplicial set

$$
\stackrel{\lim }{\longleftarrow}\left(\begin{array}{r}
\mathcal{C}_{* \times \ell, 1} \\
\downarrow(\text { src,tar }) \\
\left\{C^{\prime}\right\} \times\{C\} \longrightarrow \mathcal{C}_{* \times \ell, 0} \times \mathcal{C}_{* \times \ell, 0}
\end{array}\right) .
$$

It is clear that $\underline{\operatorname{Hom}}_{\mathcal{C}}\left(C^{\prime}, C\right)_{0}=\operatorname{Hom}_{\mathcal{C}_{\ell}}\left(C^{\prime}, C\right)$. If $d \geq 0$ and $g \in \underline{\operatorname{Hom}}_{\mathcal{C}}\left(C^{\prime}, C\right)_{d}$, then for any $\psi \in \Delta_{m}^{d}$ and $\varphi \in \Delta_{m}^{\ell}$ we have an arrow $g(\psi, \varphi)$ in $\mathcal{C}_{m}$. We compute

$$
\begin{aligned}
\operatorname{src}(g(\psi, \varphi)) & =((\psi, \varphi) \otimes 1)^{*}(\operatorname{src}(g)) \\
& =((\psi, \varphi) \otimes 1)^{*}\left(\varphi_{d}^{*}\left(C^{\prime}\right)\right) \\
& =((\psi, \varphi) \otimes 1)^{*}\left(\varphi_{d} \times 1 \otimes 1\right)^{*}\left(C^{\prime}\right) \\
& =\left(\left(\varphi_{d} \circ \psi, \varphi\right) \bigotimes 1\right)^{*}\left(C^{\prime}\right) \\
& =\left(\left(\varphi_{m}, \varphi\right) \bigotimes 1\right)^{*}\left(C^{\prime}\right)
\end{aligned}
$$

Now, identifying $\Delta^{0} \times \Delta^{\ell}$ with $\Delta^{\ell}$, we see that we may identify $\left(\varphi_{m}, \varphi\right)$ with $\varphi$, and thus

$$
\operatorname{src}(g(\psi, \varphi))=(\varphi \otimes 1)^{*}\left(C^{\prime}\right)=\varphi^{*} C^{\prime} .
$$

The same sort of computation shows that $\operatorname{tar}(g(\psi, \varphi))=\varphi^{*} C$, so that

$$
g(\psi, \varphi) \in \operatorname{Hom}_{\mathcal{C}_{m}}\left(\varphi^{*} C^{\prime}, \varphi^{*} C\right)
$$

making it clear that the $d$-simplex $g$ can be regarded as a $d$-parameter family of maps from $C^{\prime}$ to $C$.

We define $\underline{\operatorname{Isom}}_{\mathcal{C}}\left(C^{\prime}, C\right)$ to be the subsimplicial set of $\underline{\operatorname{Hom}}_{\mathcal{C}}\left(C^{\prime}, C\right)$ whose $d$-simplices are those $g$ such that $g(\psi, \varphi)$ is an isomorphism for every $m \geq 0$, every $\psi \in \Delta_{m}^{d}$, and every $\varphi \in \Delta_{m}^{\ell}$. We define $\underline{\operatorname{End}}_{\mathcal{C}}(C)=\underline{\operatorname{Hom}}_{\mathcal{C}}(C, C)$ and $\underline{\operatorname{Aut}}_{\mathcal{C}}(C)=\underline{\operatorname{Isom}}_{\mathcal{C}}(C, C)$.

Definition 6.1. Given arrows $p$ and $q$ in $\operatorname{Hom}_{\mathcal{C}_{\ell}}\left(C^{\prime}, C\right)$, we call an element

$$
g \in \underline{\operatorname{Hom}}_{\mathcal{C}}\left(C^{\prime}, C\right)_{1}
$$

a homotopy from $p$ to $q$ if $\partial_{1}^{*} g=p$ and $\partial_{0}^{*} g=q$. We say that $p$ and $q$ are homotopic if there is a chain of homotopies connecting them, i.e., they are in the same connected component of $\underline{\operatorname{Hom}}_{\mathcal{C}}\left(C^{\prime}, C\right)$.

If $\mathcal{B}$ and $\mathcal{C}$ are simplicial categories, then we let $\operatorname{Hom}(\mathcal{B}, \mathcal{C})$ denote the simplicial set whose $d$-simplices are the maps $\Delta^{d, 0} \times \mathcal{B} \stackrel{G}{\longrightarrow} \mathcal{C}$. The face maps are defined in the evident way so that $\delta^{*} G=G \circ((\delta \otimes 1) \times 1)$. It is clear that $\underline{\operatorname{Hom}}(\mathcal{B}, \mathcal{C})_{0}=\operatorname{Hom}(\mathcal{B}, \mathcal{C})$.

Now suppose $F: \mathcal{B} \rightarrow \mathcal{C}$ is a map, that $\ell \geq 0$, and that $C$ and $C^{\prime}$ are objects of $\mathcal{C}_{\ell}$. We define a map

$$
u: \underline{\operatorname{Hom}}_{\mathcal{C}}\left(C^{\prime}, C\right) \rightarrow \underline{\operatorname{Hom}}\left(C / F, C^{\prime} / F\right)
$$

which generalizes the function

$$
\begin{aligned}
\operatorname{Hom}_{\mathcal{C}}\left(C^{\prime}, C\right) & \rightarrow \operatorname{Hom}\left(C / F, C^{\prime} / F\right) \\
p & \mapsto u(p)
\end{aligned}
$$


introduced in (5.1). For a $d$-simplex $g \in \underline{\operatorname{Hom}}_{\mathcal{C}}\left(C^{\prime}, C\right)_{d}$ we define the simplicial functor $u(g)$ on objects in degree $m$ by setting

$$
u(g)(\psi,(\varphi, f, B))=(\varphi, f \circ g(\psi, \varphi), B)
$$

for any

$$
\begin{aligned}
\psi & \in \Delta_{m}^{d} \\
(\varphi, f, B) & \in \operatorname{obj}(C / F)_{m} \\
\varphi & \in \Delta_{m}^{\ell} \\
B & \in \operatorname{obj} \mathcal{B}_{m} \\
f & \in \operatorname{Hom}_{\mathcal{C}_{m}}\left(\varphi^{*} C, F B\right)
\end{aligned}
$$

To define it on arrows is easy because the arrows in $C / F$ involve composition on the other side of $f$, and we omit the details.

Now we must check that $u(g)$ is compatible with face maps $\mu:\left[m^{\prime}\right] \rightarrow[m]$, so we compute

$$
\begin{aligned}
\mu^{*}(u(g)(\psi,(\varphi, f, B))) & =\mu^{*}(\varphi, f \circ g(\psi, \varphi), B) \\
& =\left(\mu^{*} \varphi, \mu^{*}(f \circ g(\psi, \varphi)), \mu^{*} B\right) \\
& =\left(\mu^{*} \varphi, \mu^{*} f \circ \mu^{*}(g(\psi, \varphi)), \mu^{*} B\right) \\
& =\left(\mu^{*} \varphi, \mu^{*} f \circ(\mu \otimes 1)^{*}(g(\psi, \varphi)), \mu^{*} B\right) \\
& =\left(\mu^{*} \varphi, \mu^{*} f \circ(\mu \otimes 1)^{*}((\psi, \varphi) \otimes 1)^{*} g, \mu^{*} B\right) \\
& =\left(\mu^{*} \varphi, \mu^{*} f \circ((\psi \mu, \varphi \mu) \otimes 1)^{*} g, \mu^{*} B\right) \\
& =\left(\mu^{*} \varphi, \mu^{*} f \circ g\left(\mu^{*} \psi, \mu^{*} \varphi\right), \mu^{*} B\right) \\
& =u(g)\left(\mu^{*} \psi,\left(\mu^{*} \varphi, \mu^{*} f, \mu^{*} B\right)\right) \\
& =u(g)\left(\mu^{*}(\psi,(\varphi, f, B))\right) .
\end{aligned}
$$

We have thus checked that $\mu^{*}$ and $u(g)$ commute on objects. Checking it on arrows is similar and we omit the details.

Now that we've defined $u(g)$, we must check that $u$ is a simplicial map, i.e., that it commutes with any face map $\delta:[d] \rightarrow\left[d^{\prime}\right]$. So suppose we are given $g^{\prime} \in \underline{\operatorname{Hom}}_{\mathcal{C}}\left(C^{\prime}, C\right)_{d^{\prime}}$. The computation

$$
\begin{aligned}
\left(\delta^{*}\left(u\left(g^{\prime}\right)\right)\right)(\psi,(\varphi, f, B)) & =u\left(g^{\prime}\right)\left(\delta^{*} \psi,(\varphi, f, B)\right) \\
& =\left(\varphi, f \circ g\left(\delta^{*} \psi, \varphi\right), B\right) \\
& =\left(\varphi, f \circ\left(\delta^{*} g\right)(\psi, \varphi), B\right) \\
& =u\left(\delta^{*} g\right)(\psi,(\varphi, f, B))
\end{aligned}
$$

shows commutativity on objects, and commutativity on arrows is similar.

It is clear that

$$
u_{0}: \underline{\operatorname{Hom}}_{\mathcal{C}}\left(C^{\prime}, C\right)_{0} \rightarrow \underline{\operatorname{Hom}}\left(C / F, C^{\prime} / F\right)_{0}
$$

sends an arrow $p: C^{\prime} \rightarrow C$ in $\mathcal{C}_{\ell}$ to the transition map $u(p): C / F \rightarrow C^{\prime} / F$ defined in $(5.1)$. 
If $C, C^{\prime}$, and $C^{\prime \prime}$ are objects of $\mathcal{C}_{\ell}$, then there are composition operations

$$
\underline{\operatorname{Hom}}_{\mathcal{C}}\left(C^{\prime \prime}, C^{\prime}\right) \times \underline{\operatorname{Hom}}_{\mathcal{C}}\left(C^{\prime}, C\right) \rightarrow \underline{\operatorname{Hom}}_{\mathcal{C}}\left(C^{\prime \prime}, C\right)
$$

and

$$
\underline{\operatorname{Hom}}\left(C / F, C^{\prime} / F\right) \times \underline{\operatorname{Hom}}\left(C^{\prime} / F, C^{\prime \prime} / F\right) \rightarrow \underline{\operatorname{Hom}}\left(C / F, C^{\prime \prime} / F\right),
$$

and the map we have constructed respects these operations.

If $R$ is a simplicial ring, and $\mathcal{M}$ is a simplicial additive category, we say the $\mathcal{M}$ is $R$-linear if, for each $d \geq 0, \mathcal{M}_{d}$ is an $R_{d}$-linear category, and for each map $\varphi:[e] \rightarrow[d]$, each $r \in R_{d}$, and each arrow $f$ in $\mathcal{M}_{d}$, we have the equation $\varphi^{*}(r f)=\varphi^{*}(r) \varphi^{*}(f)$.

If $\mathcal{M}$ is a simplicial additive category, and $M$ and $N$ are objects of $\mathcal{M}_{\ell}$, then $\underline{\operatorname{Hom}}_{\mathcal{M}}(M, N)$ is a simplicial abelian group, which is an additive functor of both variables. It follows that

$$
\underline{\operatorname{Hom}}_{\mathcal{M}}\left(M, N_{1} \oplus N_{2}\right) \cong \underline{\operatorname{Hom}}_{\mathcal{M}}\left(M, N_{1}\right) \oplus \underline{\operatorname{Hom}}_{\mathcal{M}}\left(M, N_{2}\right),
$$

and the analogous formula holds in the other variable.

If $M$ is a simplicial abelian group, we say that $M$ is an $R$-module if there is a simplicial map $R \times M \rightarrow M$ which makes $M_{d}$ into an $R_{d}$-module for each $d$.

Assume that $\mathcal{M}$ is $R$-linear. Given $\ell \geq 0$ and objects $M$ and $N$ of $\mathcal{M}_{\ell}$, we make the simplicial abelian group $\underline{\operatorname{Hom}}_{\mathcal{M}}(M, N)$ into an $R$-module as follows. Given $d \geq 0$, $r \in R_{d}$, and $g \in \underline{\operatorname{Hom}}_{\mathcal{M}}(M, N)_{d}$ we define $r g \in \underline{\operatorname{Hom}}_{\mathcal{M}}(M, N)_{d}$ by the formula

$$
(r g)((\psi, \varphi), \beta)=(r(\psi))(g((\psi, \varphi), \beta))
$$

for $m \geq 0, \psi \in \Delta_{m}^{d}, \varphi \in \Delta_{m}^{\ell}$, and $\beta \in \Delta_{m}^{1}$.

For $n \geq 0$ we let $\operatorname{End}_{R}\left(R^{n}\right)$ denote the simplicial ring $d \mapsto \operatorname{End}_{R_{d}}\left(R_{d}^{n}\right)$. We define the simplicial group $G \ell_{n}(R)=\operatorname{Aut}_{R}\left(R^{n}\right)$ similarly.

We may use scalar multiplication to define a simplicial map

$$
R \rightarrow \underline{\operatorname{Hom}}_{\mathcal{M}}(M, M)
$$

by sending $r \in R_{d}$ to $r \cdot \varphi_{d}^{*}\left(1_{M}\right)$, where $1_{M} \in \operatorname{Hom}_{\mathcal{M}_{0}}(M, M)=\underline{\operatorname{Hom}}_{\mathcal{M}}(M, M)_{0}$. Another way to say it is that $r \cdot \varphi_{d}^{*}\left(1_{M}\right)$ sends an element $((\psi, \varphi), 1)$ in $\Delta_{m}^{d} \times \Delta_{m}^{\ell} \otimes \Delta_{1}^{1}$ to $\psi^{*}(r) \cdot 1_{\varphi^{*} M}$. We may assemble $n^{2}$ instances of this map into a map $\operatorname{End}_{R}\left(R^{n}\right) \rightarrow$ End $_{\mathcal{M}}\left(M^{n}\right)$. This map induces a map

$$
G \ell_{n}(R) \rightarrow \underline{\operatorname{Aut}}_{\mathcal{M}}\left(M^{n}\right) .
$$

\section{Loop spaces for simplicial spaces.}

Suppose now that $X$ is a pointed simplicial space. As above, we let $I_{*} X_{d}$ denote the connected component of $X_{d}$ containing the base point. One might expect that the space $\left|d \mapsto \Omega X_{d}\right|$ would be a close approximation to $\Omega|X|$, and indeed, there is a map $\left|d \mapsto \Omega X_{d}\right| \rightarrow \Omega|X|$, but there is an essential obstruction to this map being an equivalence. Observe that $\Omega I_{*} X_{d}=\Omega X_{d}$ because the loops in the loop space start at the base point, and don't wander into the other components of the space. Thus the map $\left|d \mapsto \Omega X_{d}\right| \rightarrow \Omega|X|$ factors through the map $\Omega\left|d \mapsto I_{*} X_{d}\right| \rightarrow \Omega|X|$. Since the composite map $I_{*} X_{d} \rightarrow X_{d} \rightarrow \pi_{0} X_{d}$ is constant (at the base point) we see that the composite map $\left|d \mapsto \Omega X_{d}\right| \rightarrow \Omega|X| \rightarrow \Omega\left|d \mapsto \pi_{0} X_{d}\right|$ is the constant map, so that when $\left|d \mapsto \pi_{0} X_{d}\right|$ is nontrivial, the map $\left|d \mapsto \Omega X_{d}\right| \rightarrow \Omega|X|$ might fail to be an equivalence. The following theorem says that this obstruction is the only one when $X$ is a simplicial group-like $H$-space. 
Theorem 7.1. If $X$ is a simplicial group-like H-space then the sequence

$$
\left|d \mapsto \Omega X_{d}\right| \rightarrow \Omega|X| \rightarrow \Omega\left|d \mapsto \pi_{0} X_{d}\right|
$$

is a fibration sequence.

Proof. In all the applications of this theorem to $K$-theory, $X$ is actually a simplicial loop space, i.e., there is a simplicial space $Y$ such that $X$ is homtopy equivalent (degreewise) to $\left(d \mapsto \Omega Y_{d}\right)$. We give first a simple proof adapted to that situation. Work in the category of topological spaces, or begin by making everything satisfy the Kan extension condition. We may assume that each space $Y_{d}$ is connected. There is a functorial way of producing a space $Y_{d}(1)$ with $\pi_{i} Y_{d}(1)=0$ for $i \neq 1$ and a map $Y_{d} \rightarrow Y_{d}(1)$ inducing an isomorphism on $\pi_{1}$. We may then modify $Y_{d}$ functorially so the map $g_{d}: Y_{d} \rightarrow Y_{d}(1)$ is a fibration. The path space $P Y_{d}$ is contractible, and the map $f_{d}: P Y_{d} \rightarrow Y_{d}$ is a fibration. The fibration sequence

$$
f_{d}^{-1}(*) \rightarrow\left(g_{d} f_{d}\right)^{-1}(*) \rightarrow g_{d}^{-1}(*)
$$

has connected base space, so by [17, Lemma 5.2] the sequence obtained from it by geometric realization with respect to $d$ is a fibration sequence. Since $g_{d}^{-1}(*)$ is connected, $\Omega\left|d \mapsto g_{d}^{-1}(*)\right|$ is homotopy equivalent to $\left|d \mapsto \Omega g_{d}^{-1}(*)\right|$, so we have a fibration sequence

$$
\left|d \mapsto \Omega g_{d}{ }^{-1}(*)\right| \rightarrow\left|d \mapsto f_{d}^{-1}(*)\right| \rightarrow\left|d \mapsto\left(g_{d} f_{d}\right)^{-1}(*)\right|
$$

which can be identified with

$$
\left|d \mapsto I_{*} X_{d}\right| \mapsto|X| \mapsto\left|d \mapsto \pi_{0} X_{d}\right| .
$$

We loop it once to obtain the following fibration sequence.

$$
\Omega\left|d \mapsto I_{*} X_{d}\right| \rightarrow \Omega|X| \rightarrow \Omega\left|d \mapsto \pi_{0} X_{d}\right|
$$

Since each space $I_{*} X_{d}$ is connected, it follows from the realization lemma [17, Lemma 5.2] of Waldhausen that the natural map $\left|d \mapsto \Omega I_{*} X_{d}\right| \rightarrow \Omega\left|d \mapsto I_{*} X_{d}\right|$ is a homotopy equivalence. Combining this with the equality $\Omega X_{d}=\Omega I_{*} X_{d}$ gives the desired result.

Here is our second proof, which does the general case. We refer to Theorem B.4 and exercise B.3.1 of [2], and use the notation and terminology used there. Each component of $X_{d}$ is a simple space, and for $n \geq 1$ the map

$$
\left(d \mapsto \pi_{n}\left(X_{d}\right)_{\text {free }}\right) \rightarrow\left(d \mapsto \pi_{0} X_{d}\right)
$$

is a surjective map of simplicial groups, hence is a fibration. It follows that $X$ satisfies the $\pi_{*}$-Kan condition. The simplicial group $\left(d \mapsto \pi_{0} X_{d}\right)$ also satisfies the $\pi_{*}$-Kan condition, so the cited theorem says that the square

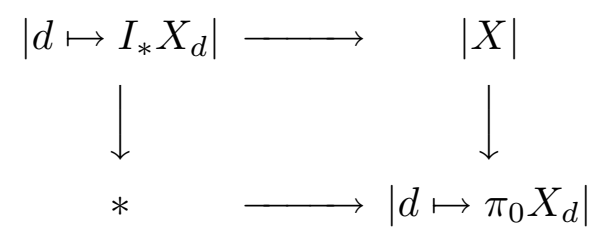


is homotopy cartesian, i.e., the sequence (7.3) is a fibration sequence. To finish, one continues as in the first proof.

The standard diagonalization technique allows us to generalize (7.1) to multisimplicial spaces. For example, if $X$ is a bisimplicial group-like $H$-space, then

$$
\left|(d, e) \mapsto \Omega X_{d, e}\right| \rightarrow \Omega|X| \rightarrow \Omega\left|(d, e) \mapsto \pi_{0} X_{d, e}\right|
$$

is a fibration sequence.

Now suppose that $X$ is a pointed simplicial set. From the discussion above, it is clear that the best one could hope for the space $\left|d \mapsto \omega X_{d}\right|$ is that it fit into the following fibration sequence.

$$
\left|d \mapsto \omega X_{d}\right| \rightarrow \Omega|X| \rightarrow \Omega\left|d \mapsto \pi_{0} X_{d}\right|,
$$

which is evidently equivalent to hoping that

$$
\left|d \mapsto \omega X_{d}\right| \rightarrow\left|d \mapsto \Omega X_{d}\right|
$$

is a homotopy equivalence. The sequence (7.5) turns out to be a fibration sequence for certain simplicial $\mathrm{H}$-spaces $X$ arising from the $K$-theory of a simplicial additive category over a connected simplicial ring, as we will see in (8.3). The H-spaces involved are actually commutative, because the operation arises from the direct sum operation for modules, so that $d \mapsto \pi_{0} X_{d}$ is a simplicial abelian group. This is the way that our fibration $W^{t+1} \rightarrow W^{t} \rightarrow W^{t} / W^{t+1}$ will arise, and this explains how natural it is for the space $W^{t} / W^{t+1}$ to be homotopy equivalent to a simplicial abelian group.

Now let's investigate what would be involved in showing that (7.5) is a fibration sequence. Comparing with (7.4), and realizing that $\omega I_{*} X_{d}=\omega X_{d}$ (because the loops in $X_{d}$ starting at the base point don't stray out of the base component), we see that it would suffice to show that the map $\left|d \mapsto \omega I_{*} X_{d}\right| \rightarrow \Omega\left|d \mapsto I_{*} X_{d}\right|$ is a homotopy equivalence. That in turn would amount to showing that

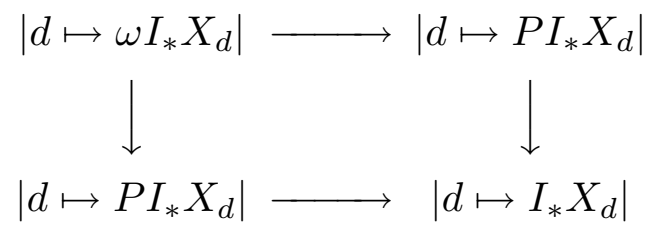

is a homotopy cartesian square. Of course, we would succeed if for each $d \geq 0$, the square

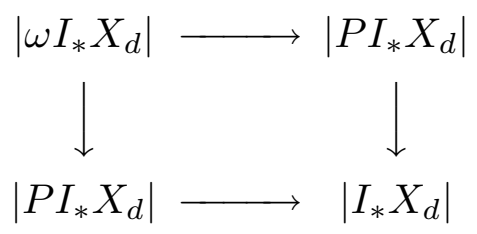

were homotopy cartesian, but in our situation, this is too much to hope for; in fact, we are introducing the simplicial direction involving $d$ because

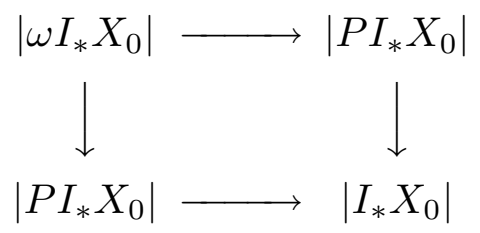


is not homotopy cartesian.

Suppose now that each $X_{d}$ is actually a category (thought of as a space via geometric realization), and $X$ is a simplicial category. The projection map

$$
\left(d \mapsto P I_{*} X_{d}\right) \stackrel{F}{\longrightarrow}\left(d \mapsto I_{*} X_{d}\right)
$$

is a map of simplicial categories, and we can apply theorem (5.3), because each category $I_{*} X_{d}$ is connected. The result is the following theorem.

Theorem 7.8. If $X$ is a simplicial category, and for all $\ell \geq 0$ the transition map $u(p)$ : $C / F \rightarrow C^{\prime} / F$ arising from any arrow $p: C^{\prime} \rightarrow C$ in $I_{*} X_{\ell}$ is a homotopy equivalence, then the sequence (7.5) is a fibration sequence.

\section{A loop space for $S^{-1} S$.}

In this section we let $R$ be a simplicial ring, and let $\mathcal{M}$ be an $R$-linear simplicial additive category. For the simplicial space $X$ of section 7 we use $X=S^{-1} S \mathcal{M}=(d \mapsto$ $\left.S^{-1} S \mathcal{M}_{d}\right)$.

Notation 8.1. Given $\ell \geq 0$ and an object $M$ of $\mathcal{M}_{\ell}$ we consider the two arrows $f_{1}, f_{2}$ : $(M, M) \rightarrow\left(M^{2}, M^{2}\right)$ in $S^{-1} S \mathcal{M}_{\ell}$ where $f_{k}=\left(i_{k}, i_{k}, 1\right)$, and where $i_{1}=\left(\begin{array}{l}1 \\ 0\end{array}\right)$ (split by $\left.p_{1}=\left(\begin{array}{ll}1 & 0\end{array}\right)\right)$, and $i_{2}=\left(\begin{array}{l}0 \\ 1\end{array}\right)$ (split by $p_{2}=\left(\begin{array}{ll}0 & 1\end{array}\right)$ ).

Lemma 8.2. With notation as above, if $R$ is contractible, then $f_{1}$ is homotopic to $f_{2}$ in the sense of (6.1).

Proof. We define $\sigma_{M}=\left(\begin{array}{cc}0 & -1_{M} \\ 1_{M} & 0\end{array}\right) \in \operatorname{Aut}\left(M^{2}\right)$ and observe that a brief calculation verifies that $f_{2}=\left(\sigma_{M}, \sigma_{M}\right) \circ f_{1}$. Indeed, $\sigma_{M} \circ i_{1}=i_{2}, p_{1} \circ \sigma_{M}{ }^{-1}=p_{2}$, and if $j$ and $i$ are composable split monomorphisms of $\mathcal{M}_{\ell}$, the composite $(j, j, 1) \circ(i, i, 1)$ in $S^{-1} S \mathcal{M}_{\ell}$ is $(j \circ j, i \circ i, 1)$.

We compose the map $\underline{\operatorname{Aut}}_{\mathcal{M}}\left(M^{2}\right) \rightarrow \underline{\mathrm{Aut}}_{S^{-1} S \mathcal{M}}\left(\left(M^{2}, M^{2}\right)\right)$ defined by $g \mapsto(g, g)$ with the map $G \ell_{2}(R) \rightarrow \underline{\operatorname{Aut}}_{\mathcal{M}}\left(M^{2}\right)$ of (6.2) to obtain a map

$$
G \ell_{2}(R) \rightarrow \text { Aut }_{S^{-1}} S \mathcal{M}\left(\left(M^{2}, M^{2}\right)\right)
$$

which sends the matrix

$$
\sigma=\left(\begin{array}{cc}
0 & -1 \\
1 & 0
\end{array}\right) \in G \ell_{2}\left(R_{0}\right)
$$

to $\left(\sigma_{M}, \sigma_{M}\right) \in \operatorname{Aut}_{S^{-1} S \mathcal{M}_{\ell}}\left(\left(M^{2}, M^{2}\right)\right)$. But $\sigma$ is the product

$$
\left(\begin{array}{cc}
1 & -1 \\
0 & 1
\end{array}\right)\left(\begin{array}{ll}
1 & 0 \\
1 & 1
\end{array}\right)\left(\begin{array}{cc}
1 & -1 \\
0 & 1
\end{array}\right)
$$

of three elementary matrices, so is connected to the identity matrix by the 1-simplex

$$
\left(\begin{array}{cc}
1 & -T \\
0 & 1
\end{array}\right)\left(\begin{array}{cc}
1 & 0 \\
T & 1
\end{array}\right)\left(\begin{array}{cc}
1 & -T \\
0 & 1
\end{array}\right)
$$

in $G \ell_{2}\left(R_{1}\right)$, where $T \in R_{1}$ connects 1 to 0 . It follows that $\left(\sigma_{M}, \sigma_{M}\right)$ is homotopic to $\left(1_{M^{2}}, 1_{M^{2}}\right)$, and thus by composition, $f_{1}$ is homotopic to $f_{2}$. 
Theorem 8.3. Let $R$ be a simplicial ring, $\mathcal{M}$ be an $R$-linear simplicial additive category, and $X=S^{-1} S \mathcal{M}$. If $R$ is contractible, then (7.5) is a fibration sequence.

Remark. An obvious prerequisite for (7.5) to be a fibration sequence is that $G=$ $\pi_{0}\left|d \mapsto \omega X_{d}\right|$ be a group. Contractibility of $R$ allows us to show $G$ is a group as follows. If $M \in \mathcal{M}_{0}$ and $\theta \in \operatorname{Aut}(M)$, then (4.4) allows us to produce a class $[M, \theta] \in G$. Then the class $\left[M, \theta^{-1}\right]$ serves as an inverse for $[M, \theta]$ because the matrix $\left(\begin{array}{cc}\theta & 0 \\ 0 & \theta^{-1}\end{array}\right)$ is a product of elementary matrices, and so is connected to the identity matrix.

Proof. In order to apply (7.8) we consider an arrow $p: C_{0} \rightarrow C_{1}$ in $I_{*} S^{-1} S \mathcal{M}_{\ell}$, and show the transition map $u(p): C_{1} / F \rightarrow C_{0} / F$ is a homotopy equivalence, where $F$ is the map in (7.7).

We write $C_{0}=\left(C_{0}^{\prime}, C_{0}^{\prime \prime}\right)$ and assume, as we may, that $C_{1}=\left(C_{0}^{\prime} \oplus M, C_{0}^{\prime \prime} \oplus M\right)$, and that $p=\left(\left(\begin{array}{l}1 \\ 0\end{array}\right),\left(\begin{array}{l}1 \\ 0\end{array}\right), 1_{M}\right)$. Direct sum with the identity stable isomorphism of $M$ with $M$ gives a map $v: C_{0} / F \rightarrow C_{1} / F$. It is enough to show that the two composites $v \circ u(p)$ and $u(p) \circ v$ are homotopic to the identity. We agree to write an object of $\left(C_{0} / F\right)_{d}$ as a pair

$$
\left(\varphi, \varphi^{*} C_{0} \rightarrow B \leftarrow(0,0)\right)
$$

where $\varphi:[d] \rightarrow[\ell]$ and $\varphi^{*} C_{0} \rightarrow B \leftarrow(0,0)$ is a diagram in $S^{-1} S \mathcal{M}_{d}$. The composite $u(p) \circ v$ is given by direct sum with

$$
(0,0) \stackrel{1_{M}}{\longrightarrow}(M, M) \stackrel{1_{M}}{\stackrel{(}{\leftrightarrows}}(0,0)
$$

so direct sum with the commutative diagram

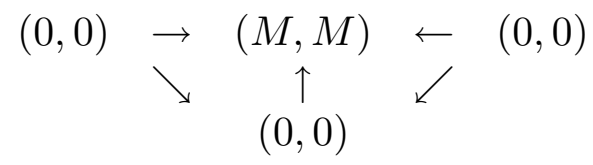

gives a homotopy from $u(p) \circ v$ to 1 .

We have a diagram

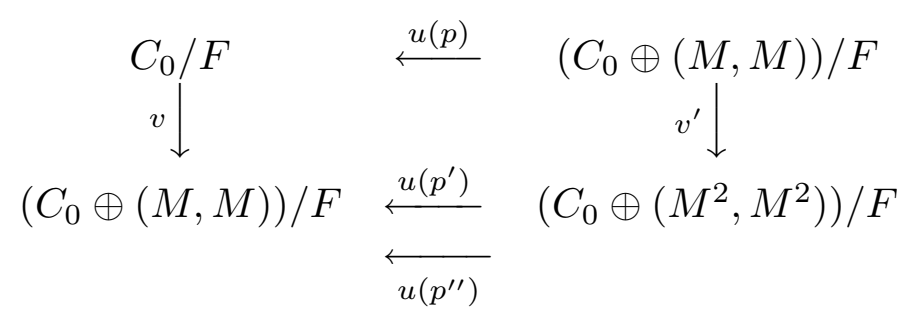

where $v^{\prime}$ is like $v$ and adds on the first $(M, M)$ factor, $p^{\prime}: C_{0} \oplus(M, M) \rightarrow C_{0} \oplus(M, M) \oplus$ $(M, M)$ is determined by the inclusion on the first $(M, M)$ factor, and $p^{\prime \prime}$ by inclusion on the second factor (with corresponding projections as splittings). The square commutes up to natural isomorphism (using $u\left(p^{\prime}\right)$ as the lower arrow), $u\left(p^{\prime}\right)$ is homotopic to $u\left(p^{\prime \prime}\right)$ (according to (8.2) and the construction of $u$ as a simplicial map in section (6)), and $u\left(p^{\prime \prime}\right) \circ v^{\prime}$ is homotopic to 1 by the same argument used above to show that $u(p) \circ v$ is homotopic to 1 . Hence $v \circ u(p)$ is homotopic to 1 . (It would have been possible to eliminate section 6 at the expense of presenting an explicit formula at this point for the homotopy between $v \circ u(p)$ and 1 , but that formula is difficult to present succinctly in printed form.) 
Remark 8.4. If $(d, e, f) \mapsto \mathcal{M}_{d e f}$ is a trisimplicial additive category, $R$ is a simplicial ring, and each $\mathcal{M}_{\text {def }}$ is $R_{d}$-linear in a natural way, then we can abuse notation and identify $R$ with the trisimplicial $\operatorname{ring}(d, e, f) \mapsto R_{d}$, allowing us to say simply that $\mathcal{M}$ is $R$-linear.

A standard diagonalization argument yields the following result.

Corollary 8.5. Let $R$ be a multisimplicial ring, $\mathcal{M}$ be an $R$-linear multisimplicial additive category. If $R$ is contractible, then the sequence

$$
\left|(d, \ldots, f) \mapsto \omega S^{-1} S \mathcal{M}_{d \ldots f}\right| \rightarrow \Omega\left|S^{-1} S \mathcal{M}\right| \rightarrow \Omega\left|(d, \ldots, f) \mapsto \pi_{0} S^{-1} S \mathcal{M}_{d \ldots f}\right|
$$

is a fibration sequence.

9. Iterated loop spaces for $S^{-1} S$.

We introduce some notation for bar resolutions. Let $G$ be a group acting on the right on a set $S$. Let $S / / G$ denote the category where an object is an element $s$ of $S$, and an arrow from $s$ to $s^{\prime}$ is an element $g \in G$ with $s g=s^{\prime}$. As usual we identify a category with its nerve. We see that $* / / G$ is the classifying space of $G$, and $G / / G$ has an initial object, so is contractible. If the action is free, then the map $S / / G \rightarrow \pi_{0}(S / / G)$ is a homotopy equivalence. If $S$ and $G$ are abelian groups written additively and the action satisfies $s+\left(s^{\prime}+g\right)=\left(s+s^{\prime}\right)+g$ then $S / / G$ is a simplicial abelian group.

Let $\mathcal{M}$ be an additive category, and let $S^{-1} S_{e} \mathcal{M}$ denote the set of $e$-simplices of the category $S^{-1} S \mathcal{M}$. We may think of $S^{-1} S_{e} \mathcal{M}$ as the set of objects of an additive category in the usual way, and use exactly the same notation to denote that category. In this way $S^{-1} S \mathcal{M}$ becomes a simplicial additive category, for which direct sum $K$-groups are defined in (4.2). The same remark applies to $e \mapsto\left(\omega S^{-1} S\right)_{e} \mathcal{M}$.

The natural map

$$
\left|e \mapsto K_{0}^{\oplus}\left(S^{-1} S_{e} \mathcal{M}\right)\right| \rightarrow K_{0}^{\oplus}\left(S^{-1} S \mathcal{M}\right)
$$

is a homotopy equivalence because the source of the map is isomorphic to the bar construction

$$
K_{0}^{\oplus} \mathcal{M} \oplus K_{0}^{\oplus} \mathcal{M} / / K_{0}^{\oplus} \mathcal{M}
$$

associated to the diagonal action (which is free), and the target of the map is

$$
\operatorname{ckr}\left(K_{0}^{\oplus}\left(S^{-1} S_{1} \mathcal{M}\right) \rightarrow K_{0}^{\oplus}\left(S^{-1} S_{0} \mathcal{M}\right)\right) .
$$

The same reasoning shows that the map

$$
\left|e \mapsto K_{0}^{\oplus}\left(\left(\omega S^{-1} S\right)_{e} \mathcal{M}\right)\right| \rightarrow K_{0}^{\oplus}\left(\omega S^{-1} S \mathcal{M}\right)
$$

is a homotopy equivalence.

Lemma 9.3. If $\mathcal{M}$ is an additive category, then the map $S^{-1} S \mathcal{M} \rightarrow\left(S^{-1} S\right)^{2} \mathcal{M}$ arising from the map obj $\mathcal{M} \rightarrow S^{-1} S \mathcal{M}$ that sends $M$ to $(M, 0)$ is a homotopy equivalence.

Proof. It is enough to show that $S^{\oplus} \mathcal{M} \rightarrow S^{\oplus}\left(S^{-1} S\right) \mathcal{M}$ is a homotopy equivalence, for $\left|S^{-1} S \mathcal{M}\right| \sim \Omega\left|S^{\oplus} \mathcal{M}\right|$. The sequence

$$
\left|e \mapsto S^{\oplus} \mathcal{M}\right| \rightarrow\left|e \mapsto S^{\oplus} S^{-1} S_{e} \mathcal{M}\right| \rightarrow\left|e \mapsto S^{\oplus} P S_{e}^{\oplus} \mathcal{M}\right|
$$

of geometric realizations of simplicial spaces is a fibration sequence (with connected base space) in each degree according to the additivity theorem, so is itself a fibration sequence. The contractibility of the base space gives the result. 
Theorem 9.4. Let $R$ be a simplicial ring, and $\mathcal{M}$ an $R$-linear simplicial additivie category. If $R$ is contractible, then the sequence

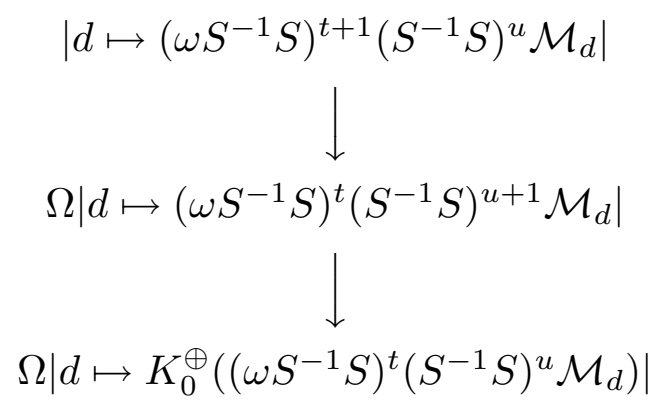

is a fibration sequence.

Proof. We apply (8.5) to the $R$-linear multisimplicial category

$$
\left(d, e_{1}, \ldots, e_{t}, f_{1}, \ldots, f_{u}\right) \mapsto\left(\omega S^{-1} S\right)_{e_{1}, \ldots, e_{t}}^{t}\left(S^{-1} S\right)_{f_{1}, \ldots, f_{u}}^{u} \mathcal{M}_{d}
$$

to get a fibration sequence whose third term can be rewritten as required according to (9.1) and (9.2).

Corollary 9.5. The homotopy type of $\left|d \mapsto\left(\omega S^{-1} S\right)^{t+1}\left(S^{-1} S\right)^{u} \mathcal{M}_{d}\right|$ is independent of $u \geq 0$.

Proof. The map used in (9.3) gives rise to a map

$$
\left|d \mapsto\left(\omega S^{-1} S\right)^{t+1}\left(S^{-1} S\right)^{u} \mathcal{M}_{d}\right| \rightarrow\left|d \mapsto\left(\omega S^{-1} S\right)^{t+1}\left(S^{-1} S\right)^{u+1} \mathcal{M}_{d}\right|
$$

which we want to show is a homotopy equivalence. This follows immediately from (9.3) if $u \geq 1$ so we may assume $u=0$. If we apply (9.4) both to the simplicial additive category $\mathcal{M}$ and to the bisimplicial additive category $S^{-1} S \mathcal{M}$ we get a map of fibration sequences which, according to (9.3), induces homotopy equivalences on the base and total spaces, hence on the fibers, yielding the result.

Corollary 9.6. There is a filtration

$$
K^{\oplus}(\mathcal{M})=W^{0} \leftarrow W^{1} \leftarrow \ldots
$$

with

$$
W^{t}=\Omega^{-t}\left|d \mapsto K^{\oplus} \mathcal{M}_{d}\left(\mathbb{G}_{m}^{\wedge t}\right)\right|
$$

and

$$
W^{t} / W^{t+1}=\Omega^{-t}\left|d \mapsto K_{0}^{\oplus} \mathcal{M}_{d}\left(\mathbb{G}_{m}^{\wedge t}\right)\right| .
$$

Proof. We use (9.4) and (9.5) to produce a fibration sequence

$$
\begin{gathered}
\left|d \mapsto S^{-1} S\left(\omega S^{-1} S\right)^{t+1} \mathcal{M}_{d}\right| \\
\downarrow \\
\quad \_\left|d \mapsto S^{-1} S\left(\omega S^{-1} S\right)^{t} \mathcal{M}_{d}\right| \\
\downarrow \\
\quad \_\left|d \mapsto K_{0}^{\oplus}\left(\left(\omega S^{-1} S\right)^{t} \mathcal{M}_{d}\right)\right|
\end{gathered}
$$


and then use (4.4) and the definition of $K^{\oplus}$ to rewrite it as

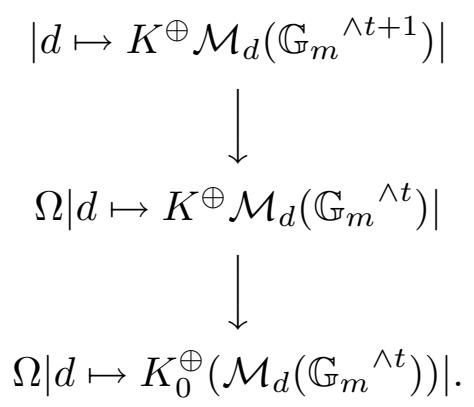

Corollary 9.7. Let $R$ be a regular noetherian ring. There is a filtration

$$
K(R) \sim W^{0} \leftarrow W^{1} \leftarrow \ldots
$$

with

$$
W^{t}=\Omega^{-t} K^{\oplus}\left(R \mathbb{A}^{\cdot}, \mathbb{G}_{m}^{\wedge t}\right)
$$

and

$$
W^{t} / W^{t+1}=\Omega^{-t}\left|d \mapsto K_{0}^{\oplus}\left(R \mathbb{A}^{d}, \mathbb{G}_{m}^{\wedge t}\right)\right|
$$

Proof. We apply (9.6) with $\mathcal{M}=\mathcal{P}\left(R \mathbb{A}^{\cdot}\right)$ and use $(2.2)$ to identify $K^{\oplus}\left(R \mathbb{A}^{*}\right) \sim K\left(R \mathbb{A}^{*}\right)$ with $K(R)$.

\section{Splitting via linear equivalence.}

In this section we show that direct-sum $K$-theory and exact sequence $K$-theory give the same result for simplicial exact categories over a connected simplicial ring $R$.

If $X$ and $Y$ are simplicial sets, we define a simplicial set $X^{Y}$ by setting $\left(X^{Y}\right)_{d}=$ $X\left(Y \times \Delta^{d}\right)=\operatorname{Hom}\left(Y \times \Delta^{d}, X\right)$. If $R$ is a simplicial ring, then we remark that $R^{Y}$ is a simplicial ring.

If $\mathcal{N}$ is an exact category, then we let $\operatorname{obj} \mathcal{N}, \operatorname{arr} \mathcal{N}$, and $\operatorname{exact} \mathcal{N}$ denote the sets of objects, arrows, and exact sequences of $\mathcal{N}$, respectively. We let $i \mathcal{N}$ denote the groupoid of isomorphisms in $\mathcal{N}$, and for $e \geq 0$ let $i_{e} \mathcal{N}$ denote the $e$-th part of its nerve. We may regard $i_{e} \mathcal{N}$ as (the objects of) an exact category in the usual way.

Lemma 10.1. Suppose $\mathcal{M}$ and $\mathcal{N}$ are exact categories, $F$ and $G$ are exact functors from $\mathcal{M}$ to $\mathcal{N}$, and $h$ is a natural isomorphism between $F$ and $G$. Let $B$ be any functor

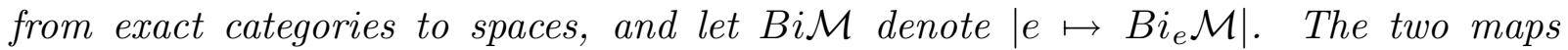
$B i F, B i G: B i \mathcal{M} \rightarrow B i \mathcal{N}$ are then connected via a homotopy provided by $h$.

Proof. It is a simple matter to convert the simplicial homotopy $i \mathcal{M}_{d} \times \Delta^{1} \rightarrow i \mathcal{N}_{d}$ provided by $h$ into a homotopy of the desired type, essentially by applying $B$.

Let $\mathcal{M}$ be a simplicial exact category. We may interpret the objects, arrows, and exact sequences of $\mathcal{M}$ as simplicial sets, denoted by obj $\mathcal{M}$, $\operatorname{arr} \mathcal{M}$, and exact $\mathcal{M}$, respectively. We let $i \mathcal{M}$ denote the simplicial groupoid of isomorphisms in $\mathcal{M}$. For any simplicial 
set $X$ we may define a category $\mathcal{M}(X)$ of diagrams whose objects, arrows, and "exact sequences" arise as follows.

$$
\begin{aligned}
\operatorname{obj}(\mathcal{M}(X)) & =\operatorname{Hom}(X, \operatorname{obj} \mathcal{M}) \\
\operatorname{arr}(\mathcal{M}(X)) & =\operatorname{Hom}(X, \operatorname{arr} \mathcal{M}) \\
\operatorname{exact}(\mathcal{M}(X)) & =\operatorname{Hom}(X, \operatorname{exact} \mathcal{M})
\end{aligned}
$$

It would be nice if $\mathcal{M}(X)$ were always an exact category, but unfortunately, this can fail to be true; it may even fail to have direct sums, because we are insisting that the maps $X \rightarrow \operatorname{obj} \mathcal{M}$ be simplicial maps (on the nose), with no isomorphisms built in. Waldhausen encountered the same problem in $[17, \mathrm{p} .180]$ for fibre products of exact categories, and solved it by building the isomorphisms into the diagram, as did Bass in [1, p. 358]. Having done that, a second problem arises, that of showing the resulting category of diagrams is an exact category, i.e., is equivalent to a full subcategory of an abelian category closed under extensions. In [17, p. 181] Waldhausen asserts that the Yoneda embeddings of the individual exact categories into abelian categories can be made functorial. Presumably something like that works here, but there is a direct artifice which we can use for the simplicial exact categories that arise in this paper. We will modify the exact categories $\mathcal{M}_{d}$ and provide them with functorial embeddings into abelian categories in such a way that the category $\mathcal{M}(X)$ (same definition as above) will be an exact category. When such a modification has been made to a simplicial exact category, we will call it an exact simplicial category.

We now describe a modification procedure that works for a typical simplicial exact category of the sort under consideration in this paper, and leave it to the reader to check that similar procedures work for the other simplicial exact categories in use here. Consider the case where $\mathcal{M}=\mathcal{P}\left(R \mathbb{A}^{\cdot}\right)$.

For a ring $R$ we consider the category $\mathcal{A}(R)$ where an object $M$ consists of (1) a collection of $S$-modules $M_{S}$, one for each $R$-algebra $S$, and (2) a collection of $S$-linear transition maps $M_{S} \rightarrow M_{T}$, one for each homomorphism $S \rightarrow T$ of $R$-algebras. These collections are subject to the requirement that an identity homomorphism of $R$-algebras yields an identity transition map, and that a commutative triangle of of $R$-algebras leads to a commutative triangle of transition maps. The arrows of $\mathcal{A}(R)$ are the evident natural transformations.

To show that a typical finite $\operatorname{limit} \lim M^{\alpha}$ exists in $\mathcal{A}(R)$ is a matter of defining $\left(\underset{\lim }{\longleftarrow} M^{\alpha}\right)_{S}=\underset{\lim }{\longleftarrow}\left(M_{S}^{\alpha}\right)$. The same method works for colimits. It follows then that $\overleftarrow{\mathcal{A}(R)}$ is an abelian category, with the evident characterization of exact sequences via exactness for each $S$. If $R \rightarrow R^{\prime}$ is a ring homomorphism, then we define an exact functor $\mathcal{A}(R) \rightarrow \mathcal{A}\left(R^{\prime}\right)$ by composing the assignment $S \mapsto M_{S}$ with the restriction functor associating an $R$-algebra to an $R^{\prime}$-algebra. Consider the full subcategory $\mathcal{P}^{\prime}(R)$ of $\mathcal{A}(R)$ whose objects are those where each transition map induces an isomorphism $M_{S} \otimes_{S} T \stackrel{\cong}{\longrightarrow} M_{T}$ and each $M_{S}$ is finitely generated and projective. The subcategory $\mathcal{P}^{\prime}(R)$ is closed under extensions in $\mathcal{A}(R)$, and is thus an exact category. The projection map $\mathcal{P}^{\prime}(R) \rightarrow \mathcal{P}(R)$ is an equivalence of categories, and thus we may replace $\mathcal{P}(R)$ by $\mathcal{P}^{\prime}(R)$ wherever required, without affecting the $K$-theory.

Consider then the situation where $\mathcal{M}=\mathcal{P}^{\prime}\left(R \mathbb{A}^{*}\right)$ and $\mathcal{A}=\mathcal{A}\left(R \mathbb{A}^{\cdot}\right)$. The main point in checking that $\mathcal{A}(X)$ is an abelian category is the construction of finite limits and 
colimits. This is easily done, provided that at the level of modules, limits are chosen so that identical limits always result from identical systems of modules. To check that $\mathcal{M}(X)$ is an exact category, one checks that $\mathcal{M}(X)$ is a full subcategory of $\mathcal{A}(X)$ closed under extensions.

This ends the discussion of the modification required to make $\mathcal{M}(X)$ into an exact category.

If $X \rightarrow Y$ is a map of simplicial sets, then there is an exact functor $\mathcal{M}(Y) \rightarrow \mathcal{M}(X)$.

If $R$ is a simplicial ring, and $\mathcal{M}$ is an $R$-linear exact simplicial category, then the category $\mathcal{M}(X)$ is $R(X)$-linear.

If $\mathcal{M}$ is an exact simplicial category, and $Y$ is a simplicial set, we define an exact simplical category $\mathcal{M}^{Y}$ by setting $\left(\mathcal{M}^{Y}\right)_{d}=\mathcal{M}\left(Y \times \Delta_{d}\right)$.

If $\mathcal{M}$ and $\mathcal{N}$ are exact simplicial categories, we say that a homotopy between exact functors $F$ and $G$ from $\mathcal{M}$ to $\mathcal{N}$ is an exact functor $h: \mathcal{M} \rightarrow \mathcal{N}^{\Delta^{1}}$ such that $F$ and $G$ are naturally isomorphic to the maps arising from $h$ via the two maps $\Delta^{0} \rightarrow \Delta^{1}$.

Lemma 10.2. Suppose $\mathcal{M}$ and $\mathcal{N}$ are exact simplicial categories, $F$ and $G$ are exact functors from $\mathcal{M}$ to $\mathcal{N}$, and $h$ is a homotopy between $F$ and $G$. Let $B$ be any functor from exact categories to spaces, and let Bi $\mathcal{M}$ denote $\left|(d, e) \mapsto B i_{e} \mathcal{M}_{d}\right|$. The two maps $B i F, B i G: B i \mathcal{M} \rightarrow B i \mathcal{N}$ are then connected via a homotopy provided by $h$.

Proof. Taking (10.1) into account, it suffices to construct a suitable map

$$
\left(B i \mathcal{N}^{\Delta^{1}}\right) \times\left|\Delta^{1}\right| \rightarrow B i \mathcal{N}
$$

We construct this by providing, for each $d \geq 0$, a map

$$
\left(\operatorname{BiN}\left(\Delta^{1} \times \Delta^{d}\right)\right) \times\left(\Delta^{1}\right)_{d} \rightarrow B i \mathcal{N}_{d} .
$$

For each $f \in\left(\Delta^{1}\right)_{d}$ we must provide a map $\operatorname{Bi\mathcal {N}}\left(\Delta^{1} \times \Delta^{d}\right) \rightarrow \operatorname{Bi\mathcal {N}}\left(\Delta^{d}\right)$, and this we obtain from the map $(1, f): \Delta^{d} \rightarrow \Delta^{1} \times \Delta^{d}$. One checks the properties required easily.

Proposition 10.3. Let $R$ be a contractible simplicial ring, $\mathcal{M}$ and $\mathcal{N}$ be $R$-linear exact simplicial categories, and $E: 0 \rightarrow F^{\prime} \rightarrow F \rightarrow F^{\prime \prime} \rightarrow 0$ be an exact sequence of functors from $\mathcal{M}$ to $\mathcal{N}$. Let $E^{\prime}$ denote the exact sequence $0 \rightarrow F^{\prime} \rightarrow F^{\prime} \oplus F^{\prime \prime} \rightarrow F^{\prime \prime} \rightarrow 0$. The exact sequences $E$ and $E^{\prime}$ are naturally homotopic through a homotopy that leaves $F^{\prime}$ and $F^{\prime \prime}$ invariant.

Proof. Let $T \in R_{1}$ be an edge connecting 1 to 0 . We interpret $T$ as an element of $\left(R^{\Delta^{1}}\right)_{0}$. (Multiplication by $T$ gives a map $R \rightarrow R^{\Delta^{1}}$ which is a contracting homotopy for $R$.)

The surjection $\Delta^{1} \rightarrow \Delta^{0}$ yields an exact functor $\mathcal{N} \rightarrow \mathcal{N}^{\Delta^{1}}$. Given an object $N \in \mathcal{N}_{d}$ we let $N[T]$ denote its image under this map. Composing this map with the functor $F$ yields an exact functor $F[T]: \mathcal{M} \rightarrow \mathcal{N}^{\Delta^{1}}$. We also have the exact sequence $E[T]: 0 \rightarrow$ $F^{\prime}[T] \rightarrow F[T] \rightarrow F^{\prime \prime}[T] \rightarrow 0$. Let $H$ denote the exact sequence of exact functors from $\mathcal{M}$ to $\mathcal{N}^{\Delta^{1}}$ obtained by pulling back $E[T]$ along the multiplication map $T: F^{\prime \prime}[T] \rightarrow F^{\prime \prime}[T]$. The exact sequence $H$ is the homotopy required, for when $T$ becomes 1 or 0 in $H$, we recover something naturally isomorphic to $E$ or $E^{\prime}$ respectively. This deformation was used by Landsburg in [12, Proposition 2.2] and [13, Lemma, p. 367] for a similar purpose.

The following additivity theorem is prompted by Waldhausen's emphasis of the additivity theorem in his work. 
Theorem 10.4. Let $R$ be a contractible simplicial ring, let $\mathcal{M}$ be an $R$-linear exact simplicial category, and let $\mathcal{E} \mathcal{M}$ be the exact simplicial category where $\mathcal{E M}_{d}$ is the category of short exact sequences in $\mathcal{M}_{d}$. Let $s$ and $q$ denote the maps $\mathcal{E} \mathcal{M} \rightarrow \mathcal{M}$ which assign to a short exact sequence the subobject and the quotient object, respectively. If $B$ is any functor from exact categories to spaces, then the map $(s, q): B i \mathcal{E M} \rightarrow B i \mathcal{M} \times B i \mathcal{M}$ is a homotopy equivalence.

Proof. Direct sum gives a map the other way, which (10.2) and (10.3) show is a homotopy inverse to $(s, q)$.

Theorem 10.5. Let $R$ be a contractible simplicial ring, and let $\mathcal{M}$ be an $R$-linear exact simplicial category. Then the map $K^{\oplus} \mathcal{M} \rightarrow K \mathcal{M}$ is a homotopy equivalence.

Proof. It suffices to check that the map $K^{\oplus} i \mathcal{M} \rightarrow K i \mathcal{M}$ is a homotopy equivalence, by [18, Corollary to Lemma 1.4.1] and its analogue for direct sum $K$-theory. It suffices to check the homotopy equivalence in each degree $n \geq 0$, where we have the map $\left|S_{n}^{\oplus} i \mathcal{M}\right| \rightarrow$ $\left|S_{n} i \mathcal{M}\right|$. This map is a homotopy equivalence because, according to (10.4) and an inductive argument, the map $\left|S_{n} i \mathcal{M}\right| \rightarrow|i \mathcal{M} \times \cdots \times i \mathcal{M}|$ is a homotopy equivalence, as is the map $\left|S_{n}^{\oplus} i \mathcal{M}\right| \rightarrow|i \mathcal{M} \times \cdots \times i \mathcal{M}|$.

Theorem 10.6. Let $R$ be a regular noetherian ring. There is a filtration

$$
K(R) \sim W^{0} \leftarrow W^{1} \leftarrow \ldots
$$

with

$$
W^{t}=\Omega^{-t} K\left(R \mathbb{A} ; \mathbb{G}_{m}^{\wedge t}\right)
$$

and

$$
W^{t} / W^{t+1}=\Omega^{-t}\left|d \mapsto K_{0}^{\oplus}\left(R \mathbb{A}^{d}, \mathbb{G}_{m}^{\wedge t}\right)\right| .
$$

Proof. In light of (9.7), it will be sufficient to show that the map

$$
K^{\oplus} C^{\oplus} \mathcal{P}\left(R \mathbb{A}^{\prime}, \mathbb{G}_{m}{ }^{\wedge t}\right) \rightarrow K C \mathcal{P}\left(R \mathbb{A} ;, \mathbb{G}_{m}{ }^{\wedge t}\right)
$$

is a homotopy equivalence. Here $C^{\oplus}$ is as defined in section 4 , and $C$ is the exact sequence version defined in $[8, \S 4]$. It suffices to look at each of the $2^{t}$ corners of the cube, at which we have a map of the form

$$
K^{\oplus} \mathcal{P}\left(R \mathbb{A}^{i}, \mathbb{G}_{m}{ }^{s}\right) \rightarrow K \mathcal{P}\left(R \mathbb{A}^{i}, \mathbb{G}_{m}{ }^{s}\right)
$$

for some $s \leq t$. That this map is a homotopy equivalence follows from (10.5) applied to the $R \mathbb{A}^{-}$-linear exact simplicial category $\mathcal{P}\left(R \mathbb{A} ;, \mathbb{G}_{m}{ }^{s}\right)$.

\section{REFERENCES}

1. Hyman Bass, Algebraic K-theory, W. A. Benjamin, Inc., New York, Amsterdam, 1968.

2. A. K. Bousfield and E. M. Friedlander, Homotopy of $\Gamma$-spaces, spectra and bisimplicial sets, Geometric Applications of Homotopy Theory II, Proceedings of a conference in Evanston, 1977 (M. G. Barratt and M. E. Mahowald, eds.), Lecture Notes in Mathematics, vol. 658, Springer, Berlin, Heidelberg, New York, 1978, pp. 80-130. 
3. Henri Gillet and Daniel R. Grayson, The loop space of the Q-construction, Illinois Journal of Mathematics 31 (1987), 574-597.

4. Daniel R. Grayson, Higher algebraic K-theory II [after Daniel Quillen], Algebraic K-theory, Evanston 1976, Lecture Notes in Mathematics 551, Springer, Berlin, Heidelberg, New York, 1976, pp. 217-240.

5. Daniel R. Grayson, The K-theory of endomorphisms, Journal of Algebra 48 (1977), 439-446.

6. Daniel R. Grayson, $K_{2}$ and the K-theory of automorphisms, Journal of Algebra 58 (1979), 1230 .

7. Daniel R. Grayson, Exact sequences in algebraic K-theory, Illinois Journal of Mathematics $\mathbf{3 1}$ (1987), 598-617.

8. Daniel R. Grayson, Adams operations on higher K-theory, K-theory 6 (1992), 97-111.

9. Daniel R. Grayson, Weight filtrations in algebraic K-theory, Motives, Proceedings of the Seattle Conference, Proceedings of Symposia in Pure Mathematics, American Mathematical Society, Providence, Rhode Island, 1993 (to appear).

10. T. Gunnarson, R. Schwänzl, R. M. Vogt, and F. Waldhausen, An un-delooped version of algebraic K-theory, Journal of Pure and Applied Algebra 79 (1992), 255-270.

11. C. Kassel, Caractère de Chern bivariant, K-theory 3 (1989), 367-400.

12. Steven E. Landsburg, Relative cycles and algebraic K-theory, American Journal of Mathematics 111 (1989), 599-632.

13. Steven E. Landsburg, K-theory and patching for categories of complexes, Duke Mathematical Journal 62 (1991), 359-384.

14. Randolph McCarthy III, Cyclic homology of an exact category, Ph.D. Thesis, Cornell University, May, 1990.

15. Daniel Quillen, Higher algebraic K-theory, Proceedings of the International Congress of Mathematicians, Vancouver 1974, vol. I, Canadian Mathematical Congress, 1975, pp. 171-176.

16. C. Soulé, Opérations en K-théorie algébrique, Canadian Journal of Mathematics 37 (1985), $488-$ 550.

17. F. Waldhausen, Algebraic K-theory of generalized free products I, II, Annals of Mathematics 108 (1978), 135-256.

18. F. Waldhausen, Algebraic K-theory of spaces, Algebraic and Geometric Topology, Proceedings of a conference at Rutgers in July, 1983, Lecture Notes in Mathematics, vol. 1126, Springer, Berlin, Heidelberg, New York, 1985, pp. 318-419.

19. Friedhelm Waldhausen, Algebraic K-theory of spaces, a manifold approach, Current Trends in Algebraic Topology, Part 1 (London, Ontario - 1981), Contemporary Mathematics 2, American Mathematical Society, Providence, Rhode Island, 1982, pp. 141-184.

20. C. Weibel, $K V$ theory of categories, Transactions of the American Mathematical Society 267 (1981), 621-635. 